\title{
Systematic Review \\ Vitamin D Supplementation and Sleep: A Systematic Review and Meta-Analysis of Intervention Studies
}

\author{
Myriam Abboud (1D \\ Department of Health Sciences, College of Natural and Health Sciences, Zayed University, Dubai, \\ United Arab Emirates; myriam.abboud@zu.ac.ae
}

check for updates

Citation: Abboud, M. Vitamin D Supplementation and Sleep: A Systematic Review and

Meta-Analysis of Intervention Studies. Nutrients 2022, 14, 1076. https: / / doi.org/10.3390/ nu14051076

Academic Editor: Luigi Barrea

Received: 3 January 2022

Accepted: 1 March 2022

Published: 3 March 2022

Publisher's Note: MDPI stays neutral with regard to jurisdictional claims in published maps and institutional affiliations.

Copyright: (C) 2022 by the author. Licensee MDPI, Basel, Switzerland. This article is an open access article distributed under the terms and conditions of the Creative Commons Attribution (CC BY) license (https:// creativecommons.org/licenses/by/ $4.0 /)$.

\begin{abstract}
Background: Vitamin D deficiency is associated with sleep disorders and poor sleep quality. Whether vitamin D supplementation (VDS) helps resolve these problems remains unclear. Objective: To systematically review the effect of VDS on sleep quantity, quality, and disorders, and perform a meta-analysis of available data. Methods: The reporting of this review followed the PRISMA statement. VDS human interventions studies that reported on sleep quality, quantity, or disorders were included. Medline, CINAHL, EMBASE, PsycInfo, the Cochrane Library, Clinicaltrials.gov, and the ICTRP were searched, in addition to the references of the included articles and previous relevant reviews, without language or time restrictions. Included studies were critically appraised, findings were narratively synthesized, and a meta-analysis was conducted. Furthermore, the overall certainty of the evidence was assessed. Results: A total of 19 studies were included (13 randomized controlled trials (RCTs), 1 opportunistic addition to an RCT, 4 pre-post studies, and 1 pre-post study analyzed as a case series); 3 RCTs were meta-analyses. The risk of bias was generally low. Pre-post studies showed a significant improvement in sleep quality with VDS. Similarly, the results of the meta-analysis revealed a statistically significant decrease in the Pittsburgh Sleep Quality Index with VDS compared with placebo (mean difference, $-2.33(95 \% \mathrm{CI},-3.09,-1.57) ; p<0.001 ; \mathrm{I}^{2}=0 \%$ ), with a moderate certainty of evidence. The results regarding the effect of VDS on sleep-related impairment, difficulty, and disorders, as well as sleepiness and restless legs syndrome, were not unanimous. Conclusions: VDS is promising in improving sleep quality; however, its effect on sleep quantity and disorders needs to be further investigated.
\end{abstract}

Keywords: vitamin D; sleep; systematic review; meta-analysis

\section{Introduction}

Inadequate sleep is a common public health problem of significant personal and societal burden [1]. Sleep disorders such as insomnia, obstructive sleep apnea (OSA), excessive daytime sleepiness (EDS) and fatigue, sleep deprivation, and restless legs syndrome (RLS) are increasingly being diagnosed in clinical practice [2]. It is estimated that $59 \%$ of young adults suffer from a sleep disorder and do not get enough sleep [3] and only $36 \%$ of this population reports being free of sleep disturbances [4]. Inadequate sleep is an underappreciated determinant of health $[5,6]$ and can lead to short-term and long-term consequences. In the short run, inadequate sleep may result in cognitive and motor performance impairments, which can lead to decreased quality of life and reduced productivity $[7,8]$. In the longer term, cumulative sleep deprivation can serve as a factor in the development and exacerbation of cardiovascular and metabolic diseases, cancer, diabetes mellitus, gastrointestinal disorders, and mental illnesses [6,9]. Thus, the economic burden of inadequate sleep is substantial, warranting urgent investment in health measures to address this issue [1].

Low vitamin D status is a prevalent condition that has been linked to a wide range of adverse health outcomes $[10,11]$. Growing evidence has demonstrated that vitamin D has a role in sleep regulation [12]. Specifically, vitamin D deficiency (VDD) can increase risk of sleep disorders and is associated with sleep difficulties, shorter sleep duration, and 
nocturnal awakenings in children and adults [13-15]. The exact mechanisms by which vitamin D regulates sleep are still far from being elucidated. Plausible theories include the presence of vitamin $\mathrm{D}$ receptors on areas of the brainstem that are known to be pacemaker cells playing an important role in sleep regulation [16,17], in addition to the potential role of vitamin D in regulating melatonin, the "sleep hormone" [18].

Hence, it is plausible that vitamin D supplementation (VDS) might have a positive effect on sleep disorders, including decreased sleep latency, improved sleep efficiency, and longer sleep duration. Preventing and managing sleep disorders or correcting them by VDS is of public health relevance, given the low cost of this intervention and its effectiveness in other therapeutic areas. Given the lack of conclusive evidence in this regard, we aim to systematically review the available literature on the effect of VDS on sleep quantity, quality, and sleep disorders, and perform a meta-analysis of the available data.

\section{Materials and Methods}

\subsection{Review Design}

A predefined protocol registered at the OSF registries (DOI: 10.17605/OSF.IO/27BD3) was followed to conduct this review. Ethical approval was not mandatory.

\subsection{Criteria for Study Inclusion}

Interventional studies, whether controlled or not, conducted on individuals not having diseases or receiving medication known to influence vitamin D metabolism, such as chronic kidney disease or liver disease, with or without sleep disturbances, including vitamin D supplementation in any form, dose, or frequency as intervention, and reporting on the prevalence or severity of sleep disorders [19], such as insomnia disorders, sleep-related breathing disorders, central disorders of hypersomnolence, circadian rhythm sleep-wake disorders, parasomnias, and sleep-related movement disorders, or sleep quantity or quality, were included.

All studies extending supplementation for a minimum of 4 weeks were included in this review to ensure adequate time for the intervention to produce an effect. In addition, controlled studies involving a placebo, or a lower dose, or different form of vitamin D were included. Finally, controlled studies including a co-intervention were included if both arms of the study received the same co-intervention.

Eligible studies were those written in any language, irrespective of publication date (i.e., no language or time limit).

Exclusion criteria included non-original studies (e.g., case reports, case series, editorials, and reviews) and studies conducted on participants with conditions (e.g., chronic kidney disease) or on medications that might have an effect on vitamin $\mathrm{D}$ metabolism (e.g., phenytoin, phenobarbital, carbamazepine, and rifampin). Finally, studies evaluating the association between vitamin D status (hypo or hypervitaminosis D) and sleep were not included.

\subsection{Search Strategy}

The author searched Medline via Ovid, the Cumulative Index to Nursing and Allied Health Literature (CINAHL) via EBSCO, EMBASE via Ovid, APA PsycInfo via Ovid, the Cochrane Library, Clinicaltrials.gov, and the International Clinical Trials Registry Platform (ICTRP). Vitamin D supplementation and sleep were the key concepts followed in the search strategy: for each concept, Medical Subject Headings (MeSH) and keywords were recorded, whereby search terms included vitamin D, cholecalciferol, ergocalciferol or calcidol, combined with sleep or insomnia. The author did not apply any language or publication date restrictions to the search. The author also hand searched the reference lists of included articles and previous relevant reviews for eligible studies. The electronic search strategy was validated by a medical information specialist and the search strategy and results for Medline, Embase, and the Chocrane Library are available in Supplementary S1. 


\subsection{Study Selection}

Studies meeting the inclusion criteria previously specified were identified by screening titles and/or abstracts from electronic scientific databases via Endnote, version X6. Full texts of potentially eligible studies were retrieved. Finally, records in the grey literature search zone were further assessed for eligibility of inclusion.

\subsection{Data Extraction}

For all eligible studies, data related to the features of the study, population groups, interventions given (type, form, and the dose of vitamin $\mathrm{D}$ in experimental groups, comparator, and duration), outcomes, and main findings were extracted and recorded in a data extraction form. When reported as $\mathrm{nmol} / \mathrm{L}$, the author converted serum $25 \mathrm{OHD}$ to $\mathrm{ng} / \mathrm{mL}$ by dividing by a factor of 2.496. The author contacted the authors of some included studies to obtain additional data, when they were not reported in the published studies.

\subsection{Quality Assessment}

The Cochrane criteria (sequence generation, allocation concealment, blinding of participants and outcome assessors, incomplete outcome data, and selective outcome reporting) were used as the tool to assess the risk of bias of RCTs included in this review [20]. Furthermore, a modified version of the Cochrane Risk of Bias tool [21] (eligibility criteria, measurement of exposure and outcome, control confounding, and follow-up) was used to assess the risk of bias of non-randomized studies. Potential sources of bias for both RCTs and non-randomized studies were graded as low, high, or unclear risk.

The Grading of Recommendations Assessment, Development, and Evaluation (GRADE) criteria (risk of bias, inconsistency, indirectness, imprecision, and publication bias) were used to assess the overall certainty of the evidence presented using GRADE Evidence Profiles developed in the GRADEpro GDT software (www.gradepro.org; accessed on 8 November 2021).

\subsection{Data Synthesis}

A narrative composite of the study findings was provided when a meta-analysis was not feasible. Furthermore, author-recorded features of the study, population group characteristics, intervention provided, comparator, and the outcome were included in this composite.

A meta-analysis was conducted when participants, treatments, and outcomes shared similar characteristics to allow pooling. Standard meta-analyses comparing VDS with placebo were performed using RevMan version 5.4 (The Cochrane Collaboration, The Nordic Cochrane Centre). A random-effects model was used for the analysis of more than two studies. The results were reported as the mean difference with $95 \%$ confidence intervals. The $\mathrm{I}^{2}$ statistic was used to assess heterogeneity among different studies.

\subsection{Quality of Reporting}

The Preferred Reporting Items for Systematic reviews and Meta-Analyses literature search extension (PRISMA-S) checklist and the PRISMA statement were followed for the literature search component [22], and the reporting of this systematic review [23].

\section{Results}

\subsection{Search Results}

Details of the search process are presented in Figure 1. Out of the 19,051 screened records, 19 studies were included in this systematic review. Out of these studies, thirteen were RCTs [24-36], one was an opportunistic addition to an established randomized, double-blind, placebo-controlled trial [37], four were pre-post studies [38-41], and one was a self-controlled before-after trial, analyzed retrospectively as a case series [42]. 


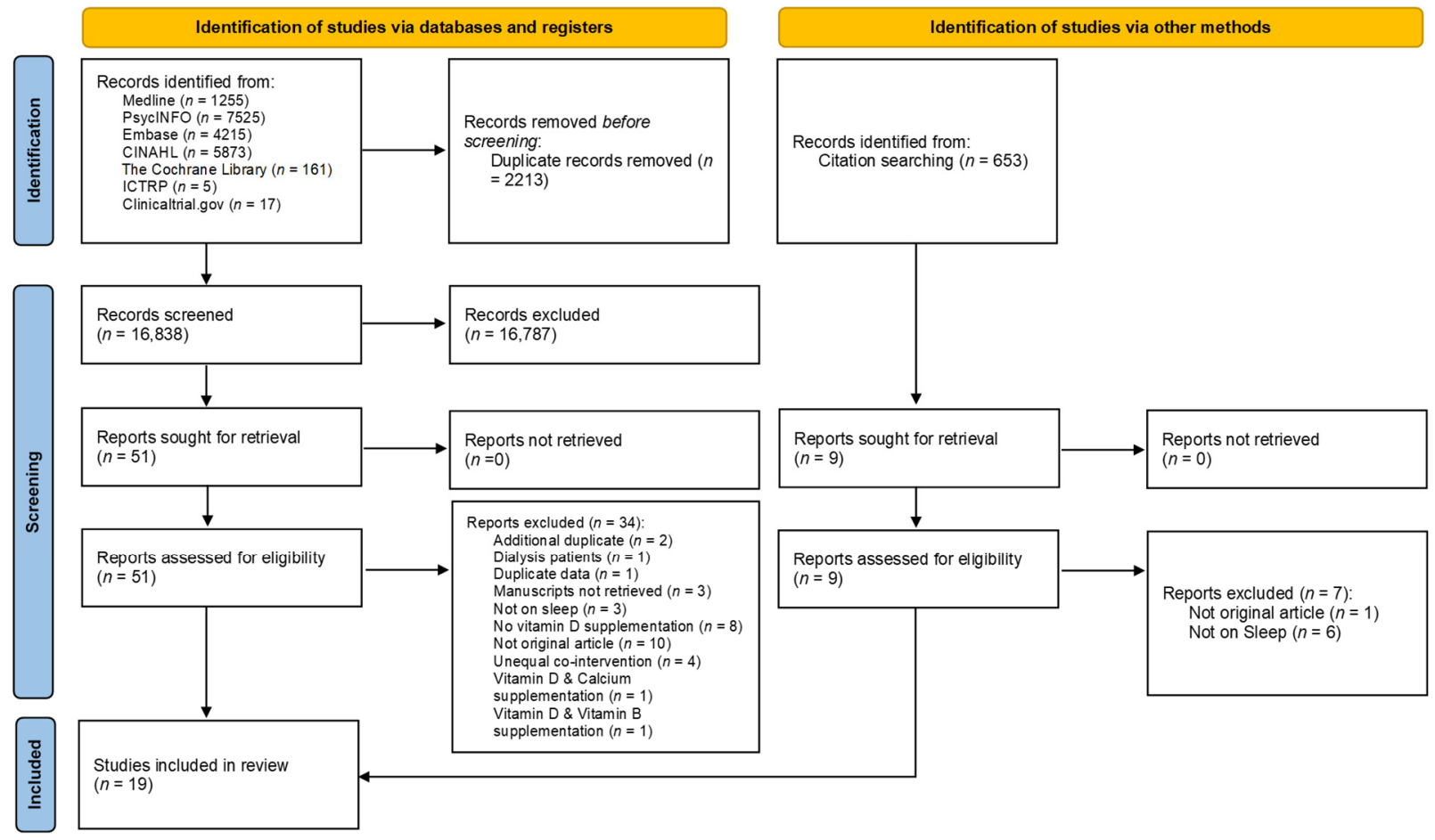

Figure 1. Preferred Reporting Items for Systematic Reviews and Meta-Analyses (PRISMA) diagram of study selection. ICTRP: International Clinical Trials Registry Platform.

Three out of thirteen RCTs generated data that could be combined in the meta-analysis.

\subsection{Characteristics of Included Studies}

Table 1 demonstrates the characteristics of the included studies. Six of the studies were conducted in Iran $[25,27,30,31,34,39]$, five in the USA $[28,29,32,33,42]$, one in Ireland [26], one in New Zealand [37], one in KSA [35], one in China [36], one in Turkey [40], and one in the Netherlands [24]. The number of participants varied from 5 [31] to 18,353 [32]. Two studies were conducted in the pediatric population: children with Autism Spectrum Disorder [40] and ADHD [31], one study was conducted on overweight postmenopausal women [28], another in elderly women [39], and one on community-dwelling older people [24]. One study was conducted in patients receiving maintenance methadone treatment [25], one in active-duty warfighters [29], one on veterans with multiple areas of chronic pain and low serum 25(OH)D [42], one on adult patients with urticarial [33], one on fibromyalgia syndrome patients [30], one in patients with chronic low back pain [41], and one on patients with depression with tied anxiety symptoms [36]. Finally, one study was conducted on adults with OSA [26], one on adults with sleep disorders [27], two in patients with RLS [35,38], and one on adults with vitamin D deficiency, abdominal obesity, and symptoms of insomnia [34].

In the majority of the studies, the intervention consisted of vitamin D3 supplementation [24,26-29,32-35,37,38,42], two used vitamin D2 supplementation [40,42], and the form of vitamin D was unclear in six trials [25,30,31,36,39,41]. Only Sharifan et al. [34] assessed VDS in the form of fortified low-fat milk or low-fat yogurt. The duration of supplementation ranged from 8 weeks $[27,30,31,39,41]$ to a median of 5.3 years [32]. The average daily dose of VDS ranged from 1000 IU $[29,39]$ to 7142.85 IU $[30,35,42]$. When reported, compliance with VDS was high in all studies. The majority of the studies were placebo controlled [24-28,30-32,35-37]. In two studies, the comparator was no supplementation [29,39]; in one study, it was a lower dose of vitamin D [33]; in one study, it was either low-fat milk or yogurt [34]. The most assessed outcome was sleep quality, mainly using the Pittsburgh Sleep Quality Index (PSQI) [25,27,28,30,39,41,42]. 
Table 1. Characteristics of included studies.

\begin{tabular}{|c|c|c|c|c|c|c|c|c|}
\hline $\begin{array}{l}\text { First Author, } \\
\text { Country }\end{array}$ & Study Design & Study Population & $\begin{array}{l}\text { Age } \\
\text { \%Male }\end{array}$ & Control & Co-Intervention & $\begin{array}{l}\text { Intervention } \\
\text { Duration } \\
\text { Daily Dose } \\
\text { Equivalent }\end{array}$ & Compliance & $\begin{array}{l}\text { Vitamin D } \\
\text { Assessment }\end{array}$ \\
\hline \multicolumn{9}{|c|}{ Randomized, controlled trial } \\
\hline \multicolumn{9}{|c|}{ Sleep quality } \\
\hline Ghaderi [25], Iran & $\begin{array}{l}\text { Randomized, } \\
\text { double-blind, } \\
\text { placebo-controlled } \\
\text { trial }\end{array}$ & $\begin{array}{l}68 \text { patients with maintenance } \\
\text { methadone treatment } \\
\text { referred to a clinic } \\
(30 \text { completed the trial: } \\
\text { I: } n=30 ; C: n=30)\end{array}$ & $\begin{array}{l}\text { Age: Range: } 25-70 \\
\text { Mean } \pm \text { SD: } \\
\text { I: } 40.1 \pm 9.2 \\
\text { C: } 42.5 \pm 8.9 \\
\text { \%Male: NR }\end{array}$ & Placebo, capsule & None & $\begin{array}{l}\text { Vitamin D (unclear): } \\
\text { capsules } \\
12 \text { weeks } \\
3571.42 \mathrm{IU}\end{array}$ & $>90 \%$ in both groups & $\begin{array}{l}\text { 25(OH)D: } \\
\text { commercial } \\
\text { ELISA kit }\end{array}$ \\
\hline $\begin{array}{l}\text { Maheshwari [41], } \\
\text { NR }\end{array}$ & Pre-post study & $\begin{array}{l}40 \text { patients diagnosed with } \\
\text { chronic low back pain }\end{array}$ & $\begin{array}{l}\text { Age: Range: } 25-65 \\
\% \text { Male: } 60 \%\end{array}$ & Self & None & $\begin{array}{l}\text { Vitamin D (unclear) } \\
8 \text { weeks } \\
\text { NR }\end{array}$ & NR & NR \\
\hline Majid [27], Iran & $\begin{array}{l}\text { Randomized, } \\
\text { double-blind, } \\
\text { placebo-controlled } \\
\text { trial }\end{array}$ & $\begin{array}{l}93 \text { people with sleep } \\
\text { disorders (PSQI } \geq 5 \text { ), but } \\
\text { without sleep disorders, not } \\
\text { using sleep medications, } \\
\text { recruited from a hospital } \\
\text { ( } 89 \text { completed the trial: } \\
\text { I: } n=44 ; \text { C: } n=45 \text { ) }\end{array}$ & $\begin{array}{l}\text { Age: Range: } 20-50 \\
\text { Mean } \pm \text { SD: } \\
\text { I: } 37.9 \pm 9.50 \\
\text { C: } 35.5 \pm 10.00 \\
\text { \%Male: I: } 25.00 ; \text { C: } \\
22.22\end{array}$ & Placebo, capsule & None & $\begin{array}{l}\text { Vitamin D3: edible } \\
\text { pearls } \\
8 \text { weeks } \\
3571.42 \text { IU }\end{array}$ & $\begin{array}{l}\text { I: } 97.82 \% \\
\text { C: } 97.87 \%\end{array}$ & $\begin{array}{l}25(\mathrm{OH}) \mathrm{D}: \\
\text { Immunodiag- } \\
\text { nostic Systems } \\
\text { kit }\end{array}$ \\
\hline Mason [28], USA & $\begin{array}{l}\text { Randomized, } \\
\text { double-blind, } \\
\text { placebo-controlled } \\
\text { trial }\end{array}$ & $\begin{array}{l}218 \text { overweight } \\
(\text { BMI } \geq 25 \mathrm{~kg} / \mathrm{m} 2) \\
\text { postmenopausal women, } \\
\text { with serum } 25(\mathrm{OH}) \mathrm{D} \geq 10 \text { to } \\
<32 \mathrm{ng} / \mathrm{mL} \text { undergoing } \\
\text { weight loss } \\
\text { I: } n=109 ; \mathrm{C}: n=109\end{array}$ & $\begin{array}{l}\text { Age: Range: } 50-75 \\
\text { Mean } \pm \text { SD: } \\
59.6 \pm 5.1 \\
\text { \%Male: } 0 \%\end{array}$ & $\begin{array}{l}\text { Placebo, gel } \\
\text { capsules }\end{array}$ & $\begin{array}{l}\text { Lifestyle-based } \\
\text { weight-loss program } \\
\text { including a } \\
\text { reduced-calorie diet } \\
(1200-2000 \mathrm{kcal} / \text { day, } \\
<30 \% \text { daily energy } \\
\text { intake from fat) and } \\
225 \mathrm{~min} / \text { week of } \\
\text { moderate-to-vigorous } \\
\text { aerobic activity }\end{array}$ & $\begin{array}{l}\text { Vitamin D3, gel } \\
\text { capsules } \\
12 \text { months } \\
2000 \mathrm{IU}\end{array}$ & $\begin{array}{l}\text { I: } 98 \% \\
\text { C: } 96 \%\end{array}$ & $\begin{array}{l}\text { 25(OH)D: } \\
\text { DiaSorin } \\
\text { LIAISON } \\
\text { 25-OH } \\
\text { Vitamin D } \\
\text { total assay }\end{array}$ \\
\hline Mirzaei [30], Iran & $\begin{array}{l}\text { Randomized, } \\
\text { double-blind, } \\
\text { placebo-controlled } \\
\text { trial }\end{array}$ & $\begin{array}{l}74 \text { fibromyalgia syndrome } \\
\text { patients (according to the } \\
\text { American College of } \\
\text { Rheumatology criteria) with } \\
25(\mathrm{OH}) \mathrm{D}<30 \mathrm{ng} / \mathrm{mL} \text {, } \\
\text { recruited from a } \\
\text { rheumatology center }\end{array}$ & $\begin{array}{l}\text { Age: Range: } 20-70 \\
\text { Mean } \pm \text { SD: } \\
\text { Total: } 41.6 \pm 10.5 \\
\text { I: } 42.1 \pm 10.8 \\
\text { C: } 41 \pm 10.3 \\
\% \text { Male: } 0 \%\end{array}$ & Placebo & $\begin{array}{l}\text { Trazodone } 25 \mathrm{mg} \text { at } \\
\text { bedtime }\end{array}$ & $\begin{array}{l}\text { Vitamin D (unclear) } \\
8 \text { weeks } \\
7142.85 \mathrm{IU}\end{array}$ & NR & $\begin{array}{l}25(\mathrm{OH}) \mathrm{D}: \\
\text { commercial } \\
\text { radioim- } \\
\text { munoassay } \\
\text { kit }\end{array}$ \\
\hline
\end{tabular}


Table 1. Cont.

\begin{tabular}{|c|c|c|c|c|c|c|c|c|}
\hline $\begin{array}{l}\text { First Author, } \\
\text { Country }\end{array}$ & Study Design & Study Population & $\begin{array}{l}\text { Age } \\
\text { \%Male }\end{array}$ & Control & Co-Intervention & $\begin{array}{l}\text { Intervention } \\
\text { Duration } \\
\text { Daily Dose } \\
\text { Equivalent }\end{array}$ & Compliance & $\begin{array}{l}\text { Vitamin D } \\
\text { Assessment }\end{array}$ \\
\hline \multicolumn{9}{|l|}{ Other outcomes } \\
\hline $\begin{array}{l}\text { McCarthy [29], } \\
\text { Pacific Northwest }\end{array}$ & $\begin{array}{l}\text { Randomized, } \\
\text { double-blind, } \\
\text { controlled trial }\end{array}$ & $\begin{array}{l}152 \text { active-duty warfighters, } \\
\text { divided into no-treatment } \\
\text { group }(\mathrm{C})(\geq 30 \mathrm{ng} / \mathrm{mL}) \text { or } \\
\text { treatment group }(\mathrm{I}) \\
(<30 \mathrm{ng} / \mathrm{mL})(131 \text { completed } \\
\text { the trial })\end{array}$ & $\begin{array}{l}\text { Age: Mean } \pm \text { SD: } \\
\text { I: } 31.6 \pm 8.2 \\
\text { C: } 32.8 \pm 10.5 \\
\% \text { Male: } 61.8 \%\end{array}$ & $\begin{array}{l}\text { No } \\
\text { supplementation }\end{array}$ & None & $\begin{array}{l}\text { Vitamin D3, gel } \\
\text { capsules } \\
12 \text { weeks } \\
\text { I1: } 1000 \mathrm{IU} \\
\text { I2: } 5000 \mathrm{IU}\end{array}$ & $\begin{array}{l}\text { I1: } 84.8 \% \\
\text { I2: } 87.9 \% \\
\text { C: not required to } \\
\text { take pills }\end{array}$ & $\begin{array}{l}\text { 25(OH)D: } \\
\text { electrochemi- } \\
\text { luminescence }\end{array}$ \\
\hline Okereke [32], USA & $\begin{array}{l}\text { Randomized, } \\
\text { double-blind, } \\
\text { placebo-controlled } \\
\text { trial }\end{array}$ & $\begin{array}{l}\text { Participants aged } \geq 50 \text { years } \\
\text { in the VITAL-DEP (Vitamin } \\
\text { D and Omega-3 } \\
\text { Trial-Depression Endpoint } \\
\text { Prevention) ancillary study } \\
\text { to VITAL, a randomized } \\
\text { clinical trial of cardiovascular } \\
\text { disease and cancer } \\
\text { prevention, without clinically } \\
\text { relevant depressive } \\
\text { symptoms at baseline } \\
\text { I: } n=9181 ; \text { C: } n=9172\end{array}$ & $\begin{array}{l}\text { Age: Mean } \pm \text { SD: } \\
\text { Total: } 67.5 \pm 7.1 \\
\text { I: } 67.5 \pm 7.0 \\
\text { C: } 67.4 \pm 7.1 \\
\% \text { Male: Total: } 51 \% \\
\text { I: } 50.6 \% ; \text { C: } 51.1 \%\end{array}$ & Placebo & None & $\begin{array}{l}\text { Vitamin D3, pills } \\
\text { Median follow-up: } \\
5.3 \text { years } \\
2000 \text { IU }\end{array}$ & $\begin{array}{l}\text { Adherence rates } \\
\text { (taking at least } \\
\text { two-thirds of pills as } \\
\text { assigned) } \\
\text { I: } \\
\text { Year 1: } 94.8 \% \\
\text { Year 2: } 92.2 \% \\
\text { Year 3: } 91.5 \% \\
\text { Year 4: } 91.4 \% \\
\text { Year 5: } 90.6 \% \\
\text { C: } \\
\text { Year 1: } 94.6 \% \\
\text { Year 2: } 92.1 \% \\
\text { Year 3: } 91.0 \% \\
\text { Year 4: } 90.5 \% \\
\text { Year 5: } 89.8 \%\end{array}$ & $\begin{array}{l}25(\mathrm{OH}) \mathrm{D}: \\
\text { radioim- } \\
\text { munoassay }\end{array}$ \\
\hline Rorie [33], USA & $\begin{array}{l}\text { Randomized, } \\
\text { double-blind, } \\
\text { controlled trial }\end{array}$ & $\begin{array}{l}42 \text { adult patients with } \\
\text { physician-diagnosed chronic } \\
\text { urticaria, recruited from } \\
\text { tertiary care clinics } \\
\text { I: } n=21 ; \text { C: } n=21\end{array}$ & $\begin{array}{l}\text { Age: } \\
\text { I: Mean: } 43.9 ; \text { Range: } \\
20-72 \\
\text { C: Mean: } 43.1 ; \\
\text { Range: } 19-79 \\
\text { \%Male: I: } 14.2 \% \text {; } \\
\text { C: } 28.5 \%\end{array}$ & $\begin{array}{l}\text { Low-dose } \\
\text { vitamin D3 } \\
\text { (600 IU/d) }\end{array}$ & $\begin{array}{l}10 \mathrm{mg} \text { of cetirizine } \\
\text { twice daily and } \\
\text { increase to } 4 \text { times } \\
\text { daily as needed, } 150 \\
\text { mg of ranitidine twice } \\
\text { daily, and } 10 \mathrm{mg} \text { of } \\
\text { montelukast daily. In } \\
\text { addition to rescue } \\
\text { prednisone use for } \\
\text { intolerable or } \\
\text { uncontrolled } \\
\text { symptoms }\end{array}$ & $\begin{array}{l}\text { Vitamin D3, pills (high } \\
\text { dose) } \\
12 \text { weeks } \\
4000 \mathrm{IU}\end{array}$ & $\begin{array}{l}\text { Excellent compliance } \\
\text { (1 subject in the low } \\
\text { vitamin D3 group } \\
\text { showed }<80 \% \\
\text { compliance) }\end{array}$ & $\begin{array}{l}\text { 25(OH)D: } \\
\text { Tandem mass } \\
\text { spectroscopy }\end{array}$ \\
\hline
\end{tabular}


Table 1. Cont.

\begin{tabular}{|c|c|c|c|c|c|c|c|c|}
\hline $\begin{array}{l}\text { First Author, } \\
\text { Country }\end{array}$ & Study Design & Study Population & $\begin{array}{l}\text { Age } \\
\% \text { Male }\end{array}$ & Control & Co-Intervention & $\begin{array}{l}\text { Intervention } \\
\text { Duration } \\
\text { Daily Dose } \\
\text { Equivalent }\end{array}$ & Compliance & $\begin{array}{l}\text { Vitamin D } \\
\text { Assessment }\end{array}$ \\
\hline Zhu [36], China & $\begin{array}{l}\text { Randomized, } \\
\text { placebo-controlled } \\
\text { trial }\end{array}$ & $\begin{array}{l}158 \text { patients with with } \\
25(\mathrm{OH}) \mathrm{D} \leq 75 \mathrm{nmol} / \mathrm{L} \text { and } \\
\text { depression with tied anxiety } \\
\text { symptoms recruited through } \\
\text { advertisements, assessed by } \\
\text { the Mini-International } \\
\text { Neuropsychiatric Interview } \\
\text { to confirm the diagnosis of } \\
\text { major depressive disorders } \\
\text { (106 completed the trial: } \\
\text { I: } n=62 ; \mathrm{C}: n=44)\end{array}$ & $\begin{array}{l}\text { Age: Mean } \pm \text { SD } \\
\text { I: } 46.3 \pm 9.7 \\
\text { C: } 43.3 \pm 13.7 \\
\text { \%Male: I: } 29 \% \text {; } \\
\text { C: } 22.7 \%\end{array}$ & Placebo & Fish oil (current intake) & $\begin{array}{l}\text { Vitamin D (unclear), } \\
\text { per os } \\
6 \text { months } \\
1600 \mathrm{IU}\end{array}$ & NR & $\begin{array}{l}25(\mathrm{OH}) \mathrm{D}: \\
\text { commercial } \\
\text { radioim- } \\
\text { munoassay } \\
\text { kit }\end{array}$ \\
\hline $\begin{array}{l}\text { Kerley [26], } \\
\text { Ireland }\end{array}$ & $\begin{array}{l}\text { Randomized, } \\
\text { double-blind, } \\
\text { placebo-controlled } \\
\text { trial }\end{array}$ & $\begin{array}{l}26 \text { Caucasian adults with } \\
\text { OSA recruited from a sleep } \\
\text { clinic } \\
\text { (19 completed the trial: } 15 \text { on } \\
\text { CPAP therapy and } 4 \text { CPAP } \\
\text { naïve: I: } n=10 ; \text { C: } n=9)\end{array}$ & $\begin{array}{l}\text { Age: } \\
\text { I: Range: } 42-71 \\
\text { Mean } \pm \text { SD: } 56 \pm 10 \\
\text { C: Range: } 32-68 \\
\text { Mean } \pm \text { SD: } 52 \pm 13 \\
\% \text { Male: I: } 89 \% \text {; C: } \\
60 \%\end{array}$ & Placebo, capsule & None & $\begin{array}{l}\text { Vitamin D3: Capsule } \\
15 \text { weeks } \\
4000 \mathrm{IU}\end{array}$ & $\begin{array}{l}93 \% \text { in both } \\
\text { groups }\end{array}$ & $\begin{array}{l}\text { Total 25(OH)D: } \\
\text { Architect } \\
\text { 25(OH)D } \\
\text { CMIA }\end{array}$ \\
\hline Sharifan [34], Iran & $\begin{array}{l}\text { Randomized, } \\
\text { triple-blind, } \\
\text { placebo-controlled } \\
\text { trial }\end{array}$ & $\begin{array}{l}29 \text { staff and students of a } \\
\text { university, aged } 30-50 \text { years, } \\
\text { with vitamin D deficiency } \\
(<30 \mathrm{ng} / \mathrm{mL}) \text {, abdominal } \\
\text { obesity (waist circumference } \\
\text { ( } \geq 94 \mathrm{~cm} \text { for men and } \geq 80 \mathrm{~cm} \\
\text { for women), and symptoms } \\
\text { of insomnia (according to } \\
\text { validated Insomnia Severity } \\
\text { Index) } \\
\text { Milk: I: } n=8 ; \text { C: } n=8 \\
\text { Yogurt: I: } n=7 ; \text { C: } n=6\end{array}$ & $\begin{array}{l}\text { Age: Mean } \pm \text { SD: } \\
43.2 \pm 6.59 \\
\text { Milk group: I: } 39.5 \pm \\
\text { 6.23; C: } 44.5 \pm 5.63 \\
\text { Yogurt group: I: } \\
47.42 \pm 6.8 ; \\
\text { C: } 41.5 \pm 5.99 \\
\text { \%Male: } \\
\text { Milk group: I: } 20 \% \text {; } \\
\text { C: } 80 \% \\
\text { Yogurt group: I: } \\
\text { 57.1\%; C: } 42.8 \% \\
\end{array}$ & $\begin{array}{l}\text { Simple low-fat } \\
\text { milk (200 mL/day) } \\
\text { Simple low-fat } \\
\text { yogurt ( } 150 \mathrm{~g} / \text { day })\end{array}$ & None & $\begin{array}{l}\text { I1: Vitamin } \\
\text { D3-fortified low-fat } \\
\text { milk containing } \\
\text { 1500 IU Nan } \\
\text { I2: Vitamin } \\
\text { D3-fortified low-fat } \\
\text { yogurt } \\
\text { 10 weeks } \\
\text { 1500 IU }\end{array}$ & NR & $\begin{array}{l}\text { 25(OH)D: } \\
\text { commercial } \\
\text { ELISA kits }\end{array}$ \\
\hline Wali [35], KSA & $\begin{array}{l}\text { Randomized, } \\
\text { double-blind, } \\
\text { placebo-controlled } \\
\text { trial }\end{array}$ & $\begin{array}{l}35 \text { patients with primary RLS } \\
\text { identified based on the RLS } \\
\text { diagnostic criteria of the } \\
\text { IRLSSG and recruited from } \\
\text { the Sleep Medicine and } \\
\text { Research Center } \\
\text { I: } n=17 ; \text { C: } n=18\end{array}$ & $\begin{array}{l}\text { Age: Mean } \pm \text { SD } \\
\text { I: } 42.7 \pm 4.7 \\
\text { C: } 42.4 \pm 5.5 \\
\% \text { Male: I: } 64.7 \% \text {; } \\
\text { C: } 72.2 \%\end{array}$ & Placebo & None & $\begin{array}{l}\text { Vitamin D3, per os } \\
12 \text { weeks } \\
7142.85 \mathrm{IU}\end{array}$ & $100 \%$ & NR \\
\hline
\end{tabular}


Table 1. Cont.

\begin{tabular}{|c|c|c|c|c|c|c|c|c|}
\hline $\begin{array}{l}\text { First Author, } \\
\text { Country }\end{array}$ & Study Design & Study Population & $\begin{array}{l}\text { Age } \\
\text { \%Male }\end{array}$ & Control & Co-Intervention & $\begin{array}{l}\text { Intervention } \\
\text { Duration } \\
\text { Daily Dose } \\
\text { Equivalent }\end{array}$ & Compliance & $\begin{array}{l}\text { Vitamin D } \\
\text { Assessment }\end{array}$ \\
\hline $\begin{array}{l}\text { de Koning [24], the } \\
\text { Netherlands }\end{array}$ & $\begin{array}{l}\text { Randomized, } \\
\text { double-blind, } \\
\text { placebo-controlled } \\
\text { trial }\end{array}$ & $\begin{array}{l}155 \text { community-dwelling } \\
\text { older people, aged } 60-80 \\
\text { years, recruited from the } \\
\text { general population or } \\
\text { through general practitioners, } \\
\text { with depressive symptoms, } \\
\text { and serum } 25(\mathrm{OH}) \mathrm{D} \text { between } \\
15 \text { and } 50 \mathrm{nmol} / \mathrm{L} \text { during } \\
\text { October-March or between } \\
15 \text { and } 70 \mathrm{nmol} / \mathrm{L} \text { during } \\
\text { April-September } \\
\text { I: } n=77 ; \mathrm{C}: n=78\end{array}$ & $\begin{array}{l}\text { Age: Median [IQR] } \\
\text { I: } 67.8 \text { [65.4-71.7] C: } \\
\text { 67.3 [63.4-72.0] } \\
\text { \%Male: I: 41.6\%; } \\
\text { C: } 43.6 \%\end{array}$ & Placebo & $\begin{array}{l}\text { Calcium tablet of } \\
500 \mathrm{mg} / \text { day in case of } \\
<2 \text { dairy } \\
\text { consumptions/day } \\
\text { Participants were } \\
\text { allowed to take a } \\
\text { (multi)VDS with a } \\
\text { maximum of } \\
400 \mathrm{IU} / \text { day in } \\
\text { addition to the study } \\
\text { tablets }\end{array}$ & $\begin{array}{l}\text { Vitamin D3, tablet } \\
12 \text { months } \\
1200 \mathrm{IU}\end{array}$ & $87.10 \%$ & $\begin{array}{l}25(\mathrm{OH}) \mathrm{D}: \\
\text { liquid chro- } \\
\text { matography } \\
\text { followed by } \\
\text { tandem mass } \\
\text { spectrometry }\end{array}$ \\
\hline $\begin{array}{l}\text { Mohammadpour } \\
\text { [31], Iran }\end{array}$ & $\begin{array}{l}\text { Randomized, } \\
\text { double-blind, } \\
\text { placebo-controlled } \\
\text { trial }\end{array}$ & $\begin{array}{l}62 \text { children with ADHD } \\
\text { (based on DSM-IV criteria), } \\
\text { aged 5-12 years, referred } \\
\text { from psychiatric centers, } \\
\text { without psychiatric nor } \\
\text { neurologic comorbidities (54 } \\
\text { completed the trial: I: } n=25 \text {; } \\
\text { C: } n=29 \text { ) }\end{array}$ & $\begin{array}{l}\text { Age: Mean } \pm \text { SD: } \\
\text { Total: } 7.87 \pm 1.61 \\
\text { I: } 7.70 \pm 1.77 \\
\text { C: } 8.03 \pm 1.44 \\
\text { \%Male: I: } 71 \% \text {; } \\
\text { C: } 77.4 \%\end{array}$ & Placebo & Methylphenidate & $\begin{array}{l}\text { Vitamin D (unclear), } \\
\text { tablet } \\
8 \text { weeks } \\
2000 \mathrm{IU}\end{array}$ & $100 \%$ & $\begin{array}{l}\text { 25(OH)D3: } \\
\text { chemilumines- } \\
\text { cence }\end{array}$ \\
\hline \multicolumn{9}{|c|}{ Opportunistic addition to a randomized, controlled trial } \\
\hline \multicolumn{9}{|l|}{ Other outcomes } \\
\hline $\begin{array}{l}\text { Slow [37], New } \\
\text { Zealand }\end{array}$ & $\begin{array}{l}\text { Opportunistic } \\
\text { addition to an } \\
\text { established } \\
\text { randomized, } \\
\text { double-blind, } \\
\text { placebo-controlled } \\
\text { trial }\end{array}$ & $\begin{array}{l}322 \text { healthy adults already } \\
\text { participating in the vitamin } \\
\text { D and acute respiratory } \\
\text { infections study (VIDARIS) } \\
\text { staff or students recruited } \\
\text { from a University. } \\
\text { ( } 308 \text { completed the trial: } \\
\text { I: } n=147 ; \text { C: } n=146 \text { ) }\end{array}$ & $\begin{array}{l}\text { Age: Range: } 18-67 \\
\text { Mean } \pm \text { SD: } \\
\text { I: } 47 \pm 10 \\
\text { C: } 48 \pm 10 \\
\% \text { Male: I: } 25 \% \\
\text { C: } 25 \%\end{array}$ & Placebo & None & $\begin{array}{l}\text { Vitamin D3, per os } \\
18 \text { months } \\
6557.37 \text { for } 2 \text { months, } \\
\text { then } 3278.68 \text { IU }\end{array}$ & NR & NR \\
\hline \multicolumn{9}{|l|}{ Pre-post study } \\
\hline Sleep quality & & & & & & & & \\
\hline
\end{tabular}


Table 1. Cont.

\begin{tabular}{|c|c|c|c|c|c|c|c|c|}
\hline $\begin{array}{l}\text { First Author, } \\
\text { Country }\end{array}$ & Study Design & Study Population & $\begin{array}{l}\text { Age } \\
\text { \%Male }\end{array}$ & Control & Co-Intervention & $\begin{array}{l}\text { Intervention } \\
\text { Duration } \\
\text { Daily Dose } \\
\text { Equivalent }\end{array}$ & Compliance & $\begin{array}{l}\text { Vitamin D } \\
\text { Assessment }\end{array}$ \\
\hline Eshaghi [39], Iran & Pre-post study & $\begin{array}{l}42 \text { elderly women referred to } \\
\text { a sports counseling center, } \\
\text { with a PSQI > 11, without } \\
\text { sleep apnea, not smoking, } \\
\text { and not taking hypnotic } \\
\text { drugs ( } 36 \text { completed the trial) }\end{array}$ & $\begin{array}{l}\text { Age: Range: } 60-70 \\
\% \text { Male: } \% \%\end{array}$ & $\begin{array}{l}\text { No } \\
\text { supplementation } \\
\text { (habitual daily } \\
\text { activities) }\end{array}$ & & $\begin{array}{l}\text { Vitamin D (unclear) } \\
8 \text { weeks } \\
1000 \mathrm{IU}\end{array}$ & NR & NR \\
\hline Guler [40], Turkey & Pre-post study & $\begin{array}{l}\text { Cases: } 60 \text { patients with ASD } \\
\text { according to DSM V criteria, } \\
\text { aged between } 4 \text { and } 10 \text { years } \\
\text { Controls: } 60 \text { age- and } \\
\text { sex-matched apparently } \\
\text { healthy children }\end{array}$ & $\begin{array}{l}\text { Age: Mean } \pm \text { SD: } \\
\text { Cases: } 7.10 \pm 1.50 \\
\text { Controls: } 6.93 \pm 1.59 \\
\text { \%Male: Cases: } 73.3 \% \text {; } \\
\text { Controls: } 65 \%\end{array}$ & & & $\begin{array}{l}\text { Vitamin D2 } \\
3 \text { months } \\
\text { Vitamin D according to } \\
\text { deficiency level: } \\
\text { I1: Participants with } \\
\text { 25(OH)D: } \\
\text { 20-29 ng/mL: } 5000 \mathrm{IU} \\
\text { I2: Participants with } \\
25(\mathrm{OH}) \mathrm{D}<20 \mathrm{ng} / \mathrm{mL}: \\
7142.86 \mathrm{IU}\end{array}$ & NR & $\begin{array}{l}25(\mathrm{OH}) \mathrm{D} \text { : } \\
\text { radioim- } \\
\text { munoassay } \\
\text { using } \\
\text { commercial } \\
\text { kits }\end{array}$ \\
\hline \multicolumn{9}{|c|}{ Pre-post study, analyzed retrospectively as a case series } \\
\hline \multicolumn{9}{|l|}{ Sleep quality } \\
\hline Huang [42], USA & $\begin{array}{l}\text { Pre-post study, } \\
\text { analyzed } \\
\text { retrospectively as a } \\
\text { case series by } \\
\text { medical record } \\
\text { review }\end{array}$ & $\begin{array}{l}46 \text { veterans with multiple } \\
\text { areas of chronic pain and low } \\
\text { serum } 25(\mathrm{OH}) \mathrm{D}(<30 \mathrm{ng} / \mathrm{mL}) \\
\text { at baseline recruited from a } \\
\text { major Veterans Affairs } \\
\text { Medical Center, divided into } \\
\text { vitamin D (1) INS: } 25(\mathrm{OH}) \mathrm{D} \text { : } \\
\text { 20-29 } \mathrm{ng} / \mathrm{mL} \text {; and (2) DEF: } \\
25(\mathrm{OH}) \mathrm{D}:<20 \mathrm{ng} / \mathrm{mL} \\
(28 \text { completed the trial: } \\
\text { INS: } n=15 ; \mathrm{DEF}: n=13)\end{array}$ & $\begin{array}{l}\text { Age: Mean } \pm \text { SD: } \\
46.2 \pm 10.8 \\
\% \text { Male: } 64.3 \%\end{array}$ & Self & None & $\begin{array}{l}\text { INS: Vitamin D3: } \\
\text { per os } \\
\text { DEF: Vitamin D2: } \\
\text { per os } \\
12 \text { weeks } \\
\text { INS: } 1200 \mathrm{IU} \\
\text { DEF: } 7142.85 \text { IU }\end{array}$ & NR & $\begin{array}{l}25(\mathrm{OH}) \mathrm{D} \text { : } \\
\text { liquid } \\
\text { chromatography- } \\
\text { mass } \\
\text { spectrometry } \\
\text { assay }\end{array}$ \\
\hline
\end{tabular}

I: intervention; C: control; PSQI: Pittsburgh Sleep Quality Index; OSA: obstructive sleep apnea; NR: not reported; INS: insufficient; DEF: deficient; VDS: vitamin D supplementation 25(OH)D: 25-hydroxyvitamin D; SD: Standard Deviation; IU: International Unit; CPAP: Continuous Positive Airways Pressure; CMIA: Chemiluminescent Microparticle Immunoassay; ELISA: Enzyme-Linked Immunosorbent Assay; RLS: restless legs syndrome; IRLSSG: International Restless Legs Syndrome Study Group; DSM: Diagnostic and Statistical Manual of Mental Disorders; ADHD: Attention Deficit Hyperactivity Disorder; IQR: Interquartile Range; ASD: Autism Spectrum Disorder. 


\subsection{Assessment of Risk of Bias}

The assessment of the risk of bias of included studies is presented in Figure 2. Regarding RCTs, the risk of bias was low, except for the study by Zhu et al. [36]. As for non-randomized trials, measurement of exposure was unclear in the studies conducted by Arico et al. [38], Eshaghi et al. [39], and Maheshwari et al. [41]. Finally, risk of bias regarding incomplete follow-up was high in the studies carried out by Arico et al. [38] and Huang et al. [42].

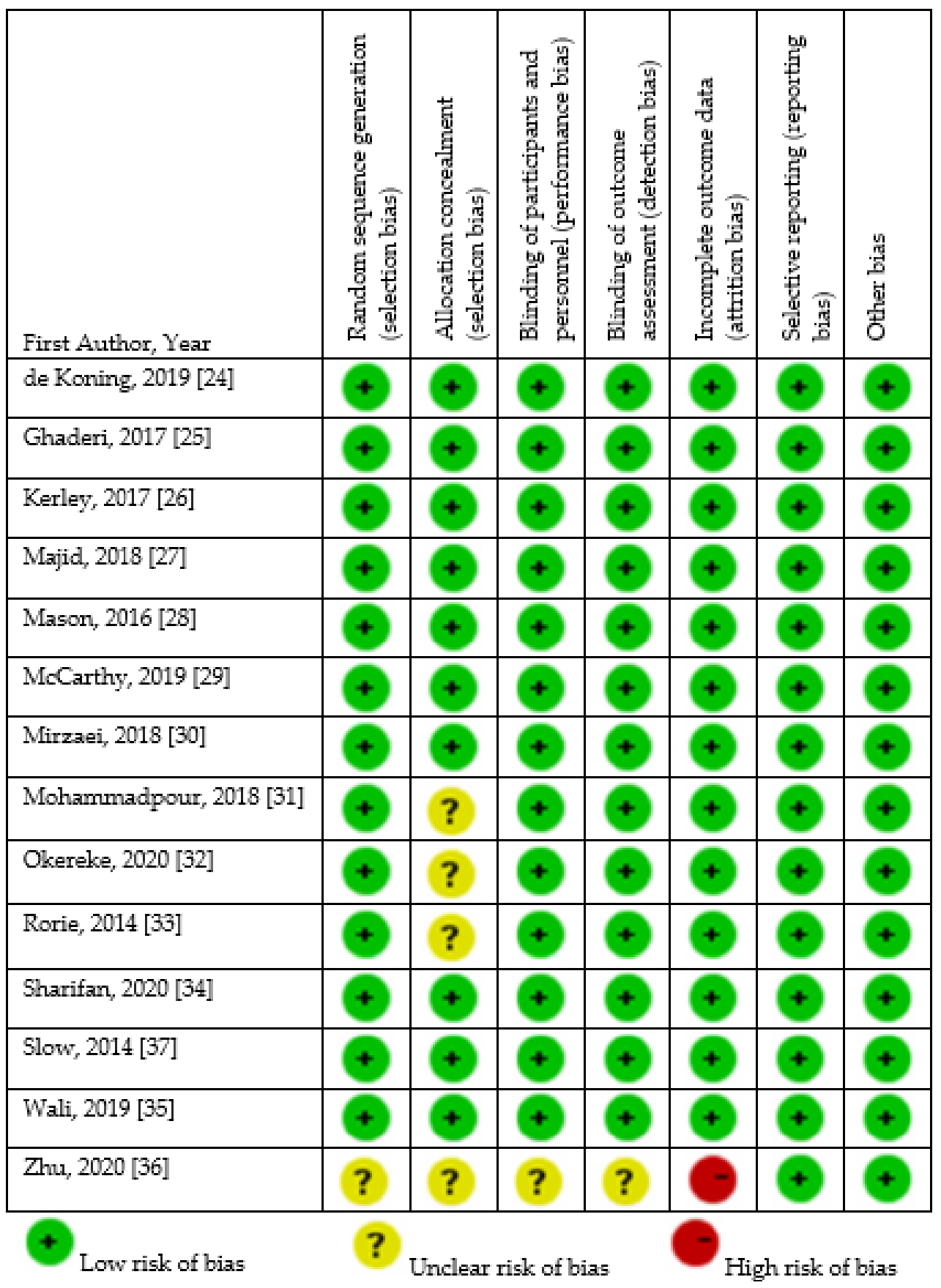

(a)

Figure 2. Cont. 


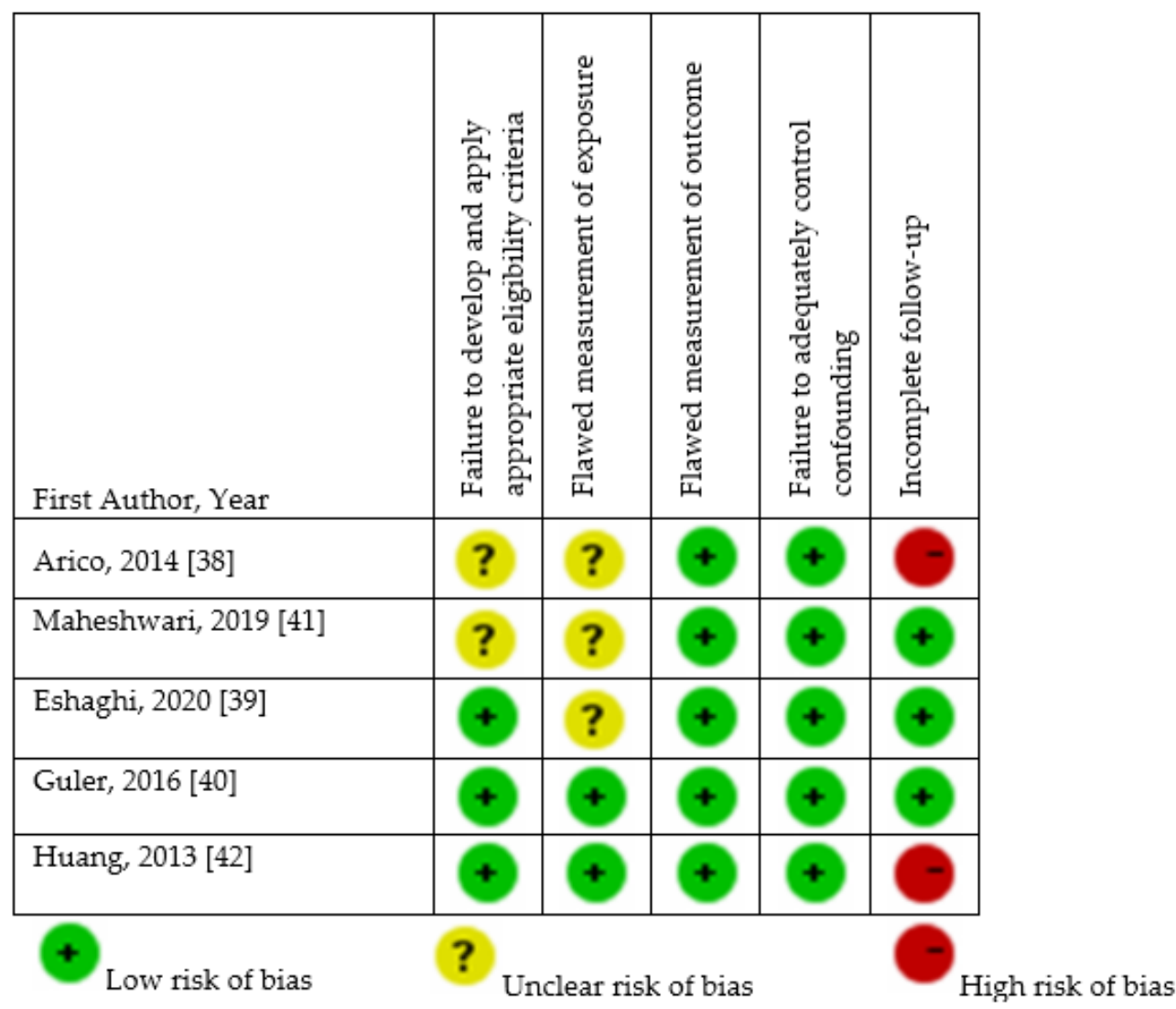

(b)

Figure 2. Risk of bias of included studies. (a). Risk of bias of included randomized controlled trials. (b). Risk of bias of included non-randomized studies [24-42].

Findings from the included studies are presented in Table 2. 
Table 2. Results of included studies.

\begin{tabular}{|c|c|c|c|c|c|c|}
\hline First Author, Country & $\begin{array}{l}\text { Outcomes Evaluated and } \\
\text { Assessment }\end{array}$ & Baseline 25OHD Level & Endline 25OHD Level & Baseline Outcomes & Endline Outcomes & Conclusion \\
\hline \multicolumn{7}{|l|}{ Randomized, controlled trial } \\
\hline \multicolumn{7}{|l|}{ Sleep quality } \\
\hline Ghaderi [25], Iran & Sleep quality: PSQI & $\begin{array}{l}\text { I: } 13.9 \pm 4.5 \\
\text { C: } 13.5 \pm 4.5 \\
\text { (NS difference between I } \\
\text { and C) }\end{array}$ & $\begin{array}{l}\text { I: } 22.0 \pm 7.5 \text { (sig. increase) } \\
\text { C: } 13.1 \pm 5.9\end{array}$ & $\begin{array}{l}\text { I: } 6.0 \pm 2.3 \\
\text { C: } 6.6 \pm 2.2\end{array}$ & $\begin{array}{l}\text { I: } 4.5 \pm 2.2 \text { (sig. decrease) } \\
\text { C: } 6.4 \pm 3.0\end{array}$ & $\begin{array}{l}\text { PSQI sig. decreased in VDS I group } \\
\text { compared with C group }(-1.5 \pm 2.2 \text { vs. } \\
-0.2 \pm 2.3)\end{array}$ \\
\hline Maheshwari [41], NR & Sleep quality: PSQI & NR & NR & NR & $\begin{array}{l}\text { t-test: } 2.965 ; \text { CI: } 1.8312-6.8341 ; p= \\
0.004 \text { (sig. differences before and } \\
\text { after VDS) }\end{array}$ & $\begin{array}{l}\text { VDS improves sleep in patients with } \\
\text { chronic low back pain }\end{array}$ \\
\hline Majid [27], Iran & $\begin{array}{l}\text { Sleep quality: PSQI } \\
\text { Sleep duration } \\
\text { Sleep latency } \\
\text { Sleep efficiency: real sleep } \\
\text { duration from the whole } \\
\text { time passed in bed } \\
\text { Sleep disturbances } \\
\text { Use of sleep medications } \\
\text { Daytime dysfunction: } \\
\text { experiencing problems } \\
\text { resulted by sleeplessness } \\
\text { Subjective sleep quality }\end{array}$ & $\begin{array}{l}\text { I: } 25.00 \pm 8.95 \\
\text { C: } 27.60 \pm 8.30 \\
\text { (NS difference between I } \\
\text { and C) }\end{array}$ & $\begin{array}{l}\text { I: } 37.69 \pm 12.25 \\
\text { C: } 27.97 \pm 7.46 \\
\text { (sig. increase in I, and sig. } \\
\text { difference between I and C) }\end{array}$ & $\begin{array}{l}\text { PSQI (score) (NS difference } \\
\text { between I and C) } \\
\text { I: } 9.45 \pm 2.44 \\
\text { C: } 10.51 \pm 3.14 \\
\text { Sleep duration (hour) (sig. higher } \\
\text { in I compared with C) } \\
\text { I: } 5.83 \pm 1.15 \\
\text { C: } 5.22 \pm 1.54 \\
\text { Sleep latency (minute) (NS } \\
\text { difference between I and C) } \\
\text { I } 49.88 \pm 38.99 \\
\text { C: } 65.00 \pm 47.54 \\
\text { Sleep efficiency (\%) (NS } \\
\text { difference between I and C) } \\
\text { I: } 82.58 \pm 9.93 \\
\text { C: } 78.20 \pm 12.90 \\
\text { Sleep disturbances (score) (NS } \\
\text { difference between I and C) } \\
\text { I: } 1.23 \pm 0.47 \\
\text { C: } 1.40 \pm 0.78 \\
\text { Use of sleep medications (time } \\
\text { per week) (NS difference between } \\
\text { I and C) } \\
\text { I: } 2.07 \pm 1.92 \\
\text { C: } 0.77 \pm 1.02 \\
\text { Day time dysfunction (score) (NS } \\
\text { difference between I and C) } \\
\text { I: } 1.57 \pm 0.99 \\
\text { C: } 1.17 \pm 0.93 \\
\text { Subjective sleep quality (score) } \\
\text { (NS difference between I and C) } \\
\text { I: } 1.68 \pm 0.77 \\
\text { C: } 1.57 \pm 0.62\end{array}$ & $\begin{array}{l}\text { PSQI (score) (sig. lower in I } \\
\text { compared with C) } \\
\text { I: } 6.75 \pm 2.97 \text { (sig. decrease) } \\
\text { C: } 9.73 \pm 3.04 \\
\text { Sleep duration (hour) (sig. higher } \\
\text { in I compared with C) } \\
\text { I: } 6.50 \pm 1.49 \\
\text { C: } 5.21 \pm 1.44 \\
\text { Sleep latency (minute) (sig. lower } \\
\text { in I compared with C) } \\
\text { I: } 33.18 \pm 27.91 \\
\text { C: } 58.57 \pm 36.81 \\
\text { Sleep efficiency (\%) (NS } \\
\text { difference between I and C) } \\
\text { I: } 86.97 \pm 11.39 \text { (sig. decrease) } \\
\text { C: } 80.89 \pm 11.46 \\
\text { Sleep disturbances (score) (NS } \\
\text { difference between I and C) } \\
\text { I: } 1.14 \pm 0.46 \text { (NS) } \\
\text { C: } 1.41 \pm 0.65 \text { (NS) } \\
\text { Use of sleep medications (time } \\
\text { per week) (NS difference between } \\
\text { I and C) } \\
\text { I: } 1.07 \pm 0.94 \text { (sig. decrease) } \\
\text { C: } 1.20 \pm 0.99 \\
\text { Day time dysfunction (score) (NS } \\
\text { difference between I and C) } \\
\text { I: } 0.70 \pm 0.96 \text { (sig. decrease) } \\
\text { C: } 0.75 \pm 0.98 \\
\text { Subjective sleep quality (score) } \\
\text { (sig. lower in I compared with C) } \\
\text { I: } 1.18 \pm 0.62 \text { (sig. decrease) } \\
\text { C: } 1.46 \pm 0.58\end{array}$ & $\begin{array}{l}\text { Reduced PSQI (improved sleep score), } \\
\text { reduced sleep latency, increased sleep } \\
\text { duration, and subjective sleep quality } \\
\text { with VDS. } \\
\text { NS difference in sleep efficiency, sleep } \\
\text { disturbances, and use of sleep } \\
\text { medications }\end{array}$ \\
\hline
\end{tabular}


Table 2. Cont.

\begin{tabular}{|c|c|c|c|c|c|c|}
\hline First Author, Country & $\begin{array}{l}\text { Outcomes Evaluated and } \\
\text { Assessment }\end{array}$ & Baseline 25OHD Level & Endline 25OHD Level & Baseline Outcomes & Endline Outcomes & Conclusion \\
\hline Mason [28], USA & Sleep quality: PSQI & NR & NR & NR & NR & $\begin{array}{l}\text { NS change in overall sleep quality } \\
\text { between VDS I and C groups } \\
\text { A greater magnitude of change in serum } \\
\text { 25(OH)D was associated with an } \\
\text { increased need to take medications to } \\
\text { sleep and overall worse sleep quality } \\
\text { Deterioration in total PSQI among } \\
\text { women who became vitamin D replete } \\
\text { ( } \geq 32 \mathrm{ng} / \mathrm{mL} \text { ) compared with those who } \\
\text { remained }<32 \mathrm{ng} / \mathrm{mL} \text { (despite VDS) } \\
\text { VDS of } 2000 \mathrm{IU} / \mathrm{d} \text { may result in overall } \\
\text { worse sleep quality for postmenopausal } \\
\text { women with low circulating vitamin D } \\
\text { undergoing weight loss }\end{array}$ \\
\hline Mirzaei [30], Iran & Sleep quality: PSQI & $\begin{array}{l}\text { I: } 11.4 \pm 6.7 \\
\text { C: } 13.4 \pm 7.3\end{array}$ & $\begin{array}{l}\text { I: } 33.5 \pm 12.2 \text { (sig. higher in I } \\
\text { compared with C) } \\
\text { C: } 13.3 \pm 7.2\end{array}$ & $\begin{array}{l}\text { I: } 10 \pm 3.3 \\
\text { C: } 10.75 \pm 4.4\end{array}$ & $\begin{array}{l}\text { I: } 6.2 \pm 2.2 \\
\text { C: } 8.2 \pm 3.7 \text { (sig. lower in I } \\
\text { compared with C) }\end{array}$ & $\begin{array}{l}\text { Considerable improvements were } \\
\text { observed in the PSQI score of the both } \\
\text { study groups; yet there was a sig. greater } \\
\text { decrease in mean PSQI score in the I } \\
\text { compared with C group }\end{array}$ \\
\hline \multicolumn{7}{|l|}{ Other outcomes } \\
\hline $\begin{array}{l}\text { McCarthy [29], Pacific } \\
\text { Northwest }\end{array}$ & $\begin{array}{l}\text { Sleep-related impairment: } \\
\text { Questions from the National } \\
\text { Institutes of Health } \\
\text { Patient-Reported Outcomes } \\
\text { Measurement Information } \\
\text { System }\end{array}$ & $\begin{array}{l}\text { I1: } 22.2 \pm 5.0 \\
\text { I2: } 22.9 \pm 4.7 \\
\text { C: } 37.8 \pm 5.6\end{array}$ & $\begin{array}{l}\text { I1: } 30.80 \pm 10.0 \\
\text { I2: } 40.15 \pm 7.5 \text { (sig. higher in } \\
\text { I2 compared with I1 and C) } \\
\text { C: } 34.46 \pm 9.9\end{array}$ & $\begin{array}{l}\text { I1: } 53.0 \pm 7.0 \\
\text { I2: } 48.3 \pm 9.5 \\
\text { C: } 51.5 \pm 7.0\end{array}$ & $\begin{array}{l}\text { I1: } 49.5 \pm 9.5 \\
\text { I2: } 45.2 \pm 8.4 \\
\text { C: } 49.3 \pm 8.2\end{array}$ & $\begin{array}{l}\text { Statistically significant improvements } \\
\text { seen across groups and over time }\end{array}$ \\
\hline Okereke [32], USA & $\begin{array}{l}\text { Sleep difficulty (sleep } \\
\text { problems) as specific } \\
\text { depressive feature } \\
\text { (item-level symptom) from } \\
\text { the } 8 \text { item Patient Health } \\
\text { Questionnaire depression } \\
\text { scale: Trouble falling or } \\
\text { staying asleep, or sleeping } \\
\text { too much }\end{array}$ & $\begin{array}{l}25(\mathrm{OH}) \mathrm{D}<20 \mathrm{ng} / \mathrm{mL} \\
\text { I: } 11.0 \% \\
\text { C: } 12.3 \% \\
\text { Mean } \pm \text { SD } \\
\text { I: } 31.2 \pm 9.8 \\
\text { C: } 31.1 \pm 10.0\end{array}$ & NR & NR & NR & $\begin{array}{l}\text { NS differences in likelihood of sleep } \\
\text { problems in the I compared with C group } \\
\text { Adjusted differences in change in } \\
\text { likelihood of PHQ-8 item-level } \\
\text { symptoms, comparing vitamin D3 to } \\
\text { Placebo: } \\
\text { Sleep problems: Likelihood ratio: } 95 \% \\
\text { CI: 1.00 (0.89-1.12) } \\
\text { Analyses were from repeated measures } \\
\text { logistic regression models, with } \\
\text { follow-up time modeled as an indicator; } \\
\text { models were controlled for age, sex, and } \\
\text { n-3 fatty acid randomization group. } \\
\text { Results show likelihood ratios and } 95 \% \\
\text { confidence intervals (95\% CIs), which } \\
\text { reflect differences in the change in } \\
\text { likelihood of burden from each PHQ-8 } \\
\text { item-level symptom, comparing vitamin } \\
\text { D3 to placebo treatment group. } \\
\text { Differences reflect the average effect over } \\
\text { all follow-up times since baseline }\end{array}$ \\
\hline
\end{tabular}


Table 2. Cont.

\begin{tabular}{|c|c|c|c|c|c|c|}
\hline First Author, Country & $\begin{array}{l}\text { Outcomes Evaluated and } \\
\text { Assessment }\end{array}$ & Baseline 25OHD Level & Endline 25OHD Level & Baseline Outcomes & Endline Outcomes & Conclusion \\
\hline Rorie [33], USA & $\begin{array}{l}\text { Nights of hives and sleep } \\
\text { interference: from the } \\
\text { Urticaria Symptom Severity } \\
\text { scores }\end{array}$ & $\begin{array}{l}\text { Mean(SE) } \\
\text { C: } 37.1(3.4) \\
\text { I: } 28.8(2.2)\end{array}$ & $\begin{array}{l}\text { Mean(SE) } \\
\text { C: } 35.8(2.3) \\
\text { I: } 56.0(3.9) \text { (sig. higher in I } \\
\text { compared with C) }\end{array}$ & NR & NR & $\begin{array}{l}\text { Beneficial trends for sleep quality and } \\
\text { towards decreased interference with } \\
\text { sleep were observed with high vitamin } \\
\text { D3 }\end{array}$ \\
\hline Zhu [36], China & Sleep disorder: NR & $\begin{array}{l}\text { I: } 15.66 \pm 4.20 \\
\text { C: } 16.86 \pm 5.04 \\
\text { (NS difference between I } \\
\text { and C group) }\end{array}$ & NR & NR & NR & $\begin{array}{l}\text { Between-group linear mixed-model } \\
\text { analysis showed sig. decrease in Sleep } \\
\text { disorder }(\beta:-0.588 ; 95 \% \mathrm{CI} \\
-1.061,-0.115) \text {, that was rendered NS } \\
\text { after controlling for confounding } \\
\text { variables }(\beta:-0.355 ; 95 \% \mathrm{CI} \text { : } \\
-0.963,0.227)\end{array}$ \\
\hline Kerley [26], Ireland & Sleepiness: ESS & $\begin{array}{l}\text { I: } 13.38 \pm 4.64 \\
\text { C: } 16.58 \pm 8.81 \\
\text { (NS difference between I } \\
\text { and C) }\end{array}$ & $\begin{array}{l}\text { I: } 40.38 \pm 15.98 \text { (sig. increase) } \\
\text { C: } 17.22 \pm 8.57\end{array}$ & $\begin{array}{l}\text { I: } 11.00 \pm 5.00 \\
\text { C: } 10.00 \pm 6.00 \\
\text { (NS difference between I and C) }\end{array}$ & $\begin{array}{l}\text { I: } 6.00 \pm 2.00 \\
\text { C: } 7.00 \pm 5.00 \\
\text { (NS difference between I and C) }\end{array}$ & $\begin{array}{l}\text { No difference in ESS between the VDS I } \\
\text { group and C group }\end{array}$ \\
\hline Sharifan [34], Iran & $\begin{array}{l}\text { Changes in sleepiness } \\
\text { symptoms: Insomnia } \\
\text { Severity Index }\end{array}$ & $\begin{array}{l}\text { Milk } \\
\text { I: } 15.03 \pm 3.91 \\
\text { C: } 14.9 \pm 7.34 \text { (NS } \\
\text { difference between I and C } \\
\text { group) } \\
\text { Yogurt } \\
\text { I: } 15.82 \pm 4.09 \\
\text { C: } 16.72 \pm 2.96 \text { (NS } \\
\text { difference between I and C } \\
\text { group) }\end{array}$ & $\begin{array}{l}\text { Milk: } \\
\text { I: } 18.57 \text { (sig. increase } \\
\text { compared with baseline) } \\
\text { C: } 14.66 \\
\text { Yogurt: } \\
\text { I: } 19.93 \text { (sig. increase } \\
\text { compared with baseline) } \\
\text { C: } 16.26 \text { (SD not reported) }\end{array}$ & $\begin{array}{l}\text { Milk: } \\
\text { I: } 18.5 \pm 3.33 \\
\text { C: } 17.25 \pm 2.34 \\
\text { Yogurt: } \\
\text { I: } 13.28 \pm 5.12 \\
\text { C: } 13 \pm 3.54\end{array}$ & $\begin{array}{l}\text { Milk } \\
\text { I: } 13.62 \pm 3.29 \text { (sig. increase } \\
\text { compared with baseline) } \\
\text { C: } 16.5 \pm 4.02 \text { (NS difference } \\
\text { compared with baseline) } \\
\text { Yogurt } \\
\text { I: } 17.57 \pm 13.28 \text { (NS difference } \\
\text { compared with baseline) } \\
\text { C: } 16.66 \pm 1.36 \text { (NS difference } \\
\text { compared with baseline) }\end{array}$ & $\begin{array}{l}\text { Fortified low-fat milk containing } 1500 \mathrm{IU} \\
\text { vitamin D3 can improve insomnia } \\
\text { symptoms }\end{array}$ \\
\hline Wali [35], KSA & $\begin{array}{l}\text { RLS severity: IRLSSG rating } \\
\text { scale }\end{array}$ & $\begin{array}{l}\text { I: } 17.06 \pm 12.6 \\
\text { C: } 22.95 \pm 16.98 \\
\text { (NS difference between I } \\
\text { and C group) }\end{array}$ & $\begin{array}{l}\text { I: } 6.09 \pm 15.38 \text { (sig. higher in } \\
\text { I compared with C group) } \\
\text { C: } 21.23 \pm 13.74\end{array}$ & $\begin{array}{l}\text { Total } \\
\text { I: } 14.60 \pm 4.5 \\
\text { C: } 16.11 \pm 6.2 \\
\text { In DEF patients } \\
\text { I: } 14.82 \pm 5.2 \\
\text { C: } 16.81 \pm 6.3\end{array}$ & $\begin{array}{l}\text { Total: } \\
\text { I: } 14.5 \pm 08.2 \text { (NS difference } \\
\text { compared with baseline) } \\
\text { C: } 10.3 \pm 11.1 \text { (sig. decrease } \\
\text { compared with baseline) } \\
\text { In DEF patients } \\
\text { I: } 13.8 \pm 8.9 \text { (NS difference } \\
\text { compared with baseline) } \\
\text { C: } 7.8 \pm 13.9 \text { (sig. decrease } \\
\text { compared with baseline) }\end{array}$ & $\begin{array}{l}\text { NS difference in RLS severity score with } \\
\text { VDS suggesting that VDS does not } \\
\text { improve RLS symptoms }\end{array}$ \\
\hline \multicolumn{7}{|c|}{ Sleep problems as adverse events of VDS } \\
\hline $\begin{array}{l}\text { de Koning [24], the } \\
\text { Netherlands }\end{array}$ & $\begin{array}{l}\text { Sleep problems as adverse } \\
\text { event: registered by } \\
\text { telephone or face-to-face } \\
\text { contact }\end{array}$ & $\begin{array}{l}\text { median [IQR] } \\
\text { I: } 18.42[13.02-22.83] \\
\text { C: } 17.68[14.42-22.13] \\
\text { (significance NR) }\end{array}$ & $\begin{array}{l}\text { mean } \pm \text { SD } \\
\text { I: } 34.05 \pm 6.41 \\
\text { C: } 17.22 \pm 7.21 \\
\text { (sig. difference between I and } \\
\text { C) }\end{array}$ & $\begin{array}{l}\text { NA (sleep problems as adverse } \\
\text { event of VDS) }\end{array}$ & $\begin{array}{l}\text { I: } n=1 \\
\text { C: } n=5 \\
\text { (NS difference between I and C) }\end{array}$ & $\begin{array}{l}\text { NS difference in sleep problems as } \\
\text { adverse effect of VDS in } \\
\text { community-dwelling people with } \\
\text { depressive symptoms }\end{array}$ \\
\hline
\end{tabular}


Table 2. Cont.

\begin{tabular}{|c|c|c|c|c|c|c|}
\hline First Author, Country & $\begin{array}{l}\text { Outcomes Evaluated and } \\
\text { Assessment }\end{array}$ & Baseline 25OHD Level & Endline 25OHD Level & Baseline Outcomes & Endline Outcomes & Conclusion \\
\hline Mohammadpour [31], Iran & $\begin{array}{l}\text { Sleep problems as adverse } \\
\text { events: questionnaire }\end{array}$ & $\begin{array}{l}\text { mean } \pm \text { SD } \\
\text { I: } 15.792 \pm 5.259 \text { C: } 12.979 \\
\pm 5.804 \text { (NS difference } \\
\text { between I and C) }\end{array}$ & $\begin{array}{l}\text { mean } \pm \text { SD: I: } 34.63 \pm 9.54 \\
\text { (sig. increase between } \\
\text { baseline and endline) } \\
\text { C: } 11.22 \pm 5.11 \text { (NS } \\
\text { difference between baseline } \\
\text { and endline) } \\
\text { Sig. difference between I } \\
\text { and C }\end{array}$ & $\begin{array}{l}\text { NA (sleep problems as adverse } \\
\text { event of VDS) }\end{array}$ & $\begin{array}{l}\text { Rate } \\
\text { I: } 4 \\
\text { C: } 1 \\
\text { (NS difference between I and C) }\end{array}$ & $\begin{array}{l}\text { NS difference in sleep problems as } \\
\text { adverse effect of VDS as adjunctive } \\
\text { therapy in children with ADHD }\end{array}$ \\
\hline \multicolumn{7}{|c|}{ Opportunistic addition to a randomized, controlled trial } \\
\hline \multicolumn{7}{|c|}{ Other outcomes } \\
\hline Slow [37], New Zealand & $\begin{array}{l}\text { Specific questionnaire about } \\
\text { disruptions in sleeping } \\
\text { patterns as a consequence of } \\
\text { the earthquake on the 22nd } \\
\text { February } 2011\end{array}$ & $\begin{array}{l}\text { I: } 29.24 \pm 8.81 \\
\text { C: } 28.44 \pm 8.81\end{array}$ & NR & NR & $\begin{array}{l}\text { I: } 79 \% \\
\text { C: } 70 \% \text { (NS difference between I } \\
\text { and C) }\end{array}$ & $\begin{array}{l}\text { VDS did not reduce the adverse impact } \\
\text { of earthquakes in healthy adults }\end{array}$ \\
\hline \multicolumn{7}{|l|}{ Pre-post study } \\
\hline \multicolumn{7}{|l|}{ Sleep quality } \\
\hline Eshaghi [39], Iran & Sleep quality: PSQI & NR & NR & $\begin{array}{l}\text { Mean } \pm \text { SD } \\
\text { I: } 12.55 \pm 1.01 \\
\text { C: } 12.55 \pm 1.01\end{array}$ & $\begin{array}{l}\text { Mean } \pm \text { SD I: } 10.11 \pm 1.26 \\
\text { (significant difference compared } \\
\text { with baseline) } \\
C: 12.44 \pm 0.88 \text { (contradictory } \\
\text { evidence regarding C group } \\
\text { between written (NS difference) } \\
\text { and tabulated (sig. } \\
\text { difference) results) }\end{array}$ & $\begin{array}{l}\text { Sleep quality sig. improved by } 19.1 \% \\
\text { with VDS }\end{array}$ \\
\hline \multicolumn{7}{|l|}{ Other outcomes } \\
\hline & $\begin{array}{l}\text { Sleep habits and disorders: } \\
\text { short version of the CSHQ }\end{array}$ & $\begin{array}{l}\text { Cases: } \\
25(\mathrm{OH}) \mathrm{D}<10 \mathrm{ng} / \mathrm{mL}: \\
23.3 \% \\
25(\mathrm{OH}) \mathrm{D}: 10-30 \mathrm{ng} / \mathrm{mL}: \\
45 \% \\
25(\mathrm{OH}) \mathrm{D}>30 \mathrm{ng} / \mathrm{mL}: \\
31.7 \% \\
\text { mean } \pm \text { SD: } 25.58 \pm 10.31 \\
\text { Controls: } \\
25(\mathrm{OH}) \mathrm{D}<10 \mathrm{ng} / \mathrm{mL}:\end{array}$ & $\begin{array}{l}\text { Cases: } \\
25(\mathrm{OH}) \mathrm{D}<10 \mathrm{ng} / \mathrm{mL}: 0 \% \\
25(\mathrm{OH}) \mathrm{D}: 10-30 \mathrm{ng} / \mathrm{mL}: \\
11.7 \% \\
25(\mathrm{OH}) \mathrm{D}>30 \mathrm{ng} / \mathrm{mL}): 88.3 \% \\
\text { mean } \pm \text { SD: } 37.27 \pm 6.51 \\
\text { Controls: } \\
25(\mathrm{OH}) \mathrm{D}<10 \mathrm{ng} / \mathrm{mL}: 0 \%\end{array}$ & $\begin{array}{l}\text { CSHQ total score } \\
\text { Cases: }<41: 21.7 \% ; \geq 41: 78.3 \% \\
\text { Controls: }<41: 66.7 \% ; \geq 41: 33.3 \% \\
\text { mean } \pm \text { SD total sleep time } \\
\text { (hours) } \\
\text { Cases: } 8.10 \pm 0.97 \\
\text { Controls: } 9.24 \pm 0.89 \\
\text { In DEF participants } \\
\text { Total score } \\
\text { Cases: } 52.05 \pm 8.24 \\
\text { Controls: } 42.00 \pm 4.78 \\
\text { Bedtime resistance } \\
\text { Cases: } 11.24 \pm 2.49 \\
\text { Controls: } 7.38 \pm 1.01 \\
\text { Sleep-onset delay } \\
\text { Cases: } 2.32 \pm 0.79 \\
\text { Controls: } 1.86 \pm 0.89 \\
\text { Sleep duration }\end{array}$ & $\begin{array}{l}\text { CSHQ total score } \\
\text { Cases: }<41: 28.3 \% ; \geq 41: 71.7 \% \\
\text { Controls: }<41: 86.7 \% ; \geq 41: 13.3 \% \\
\text { mean } \pm \text { SD total sleep time } \\
\text { (hours) } \\
\text { Cases: } 8.58 \pm 0.96 \\
\text { Controls: } 9.38 \pm 0.88 \\
\text { (sleep time sig. different between } \\
\text { Cases and Controls) } \\
\text { In DEF participants } \\
\text { Total score } \\
\text { Cases: } 46.43 \pm 8.04 \text {; sig } \\
\text { Controls: } 37.56 \pm 2.80 \text {; sig } \\
\text { Bedtime resistance } \\
\text { Cases: } 10.17 \pm 2.66 \text {; sig } \\
\text { Controls: } 7.21 \pm 0.75 ; \mathrm{NS} \\
\text { Sleep-onset delay } \\
\text { Cases: } 1.82 \pm 0.80 ; \text { sig } \\
\text { Controls: } 1.08 \pm 0.36 \text {; sig }\end{array}$ & \\
\hline
\end{tabular}


Table 2. Cont.

\begin{tabular}{|c|c|c|c|c|c|c|}
\hline First Author, Country & $\begin{array}{l}\text { Outcomes Evaluated and } \\
\text { Assessment }\end{array}$ & Baseline 25OHD Level & Endline 25OHD Level & Baseline Outcomes & Endline Outcomes & Conclusion \\
\hline Guler [40], Turkey & $\begin{array}{l}\text { with a higher score reflecting } \\
\text { more disturbed sleep } \\
\text { behavior }\end{array}$ & $\begin{array}{l}23.3 \% \\
25(\mathrm{OH}) \mathrm{D}: 10-30 \mathrm{ng} / \mathrm{mL}: \\
38.3 \% \\
25(\mathrm{OH}) \mathrm{D}>30 \mathrm{ng} / \mathrm{mL}: \\
38.3 \% \\
\text { mean } \pm \text { SD: } 25.35 \pm 9.92 \\
\text { (NS difference between } \\
\text { Cases and Controls) } \\
\text { In DEF participants: } \\
\text { 25(OH)D: } \\
\text { Cases: } 19.68 \pm 6.22 \\
\text { Controls: } 19.21 \pm 7.35\end{array}$ & $\begin{array}{l}\text { 25(OH)D: } 10-30 \mathrm{ng} / \mathrm{mL}: 6.7 \% \\
25(\mathrm{OH}) \mathrm{D}>30 \mathrm{ng} / \mathrm{mL}: 93.3 \% \\
\text { mean } \pm \text { SD: } 37.15 \pm 6.78 \\
\text { (NS difference between } \\
\text { Cases and Controls) } \\
\text { In DEF participants: } \\
\text { 25(OH)D: } \\
\text { Cases: } 37.26 \pm 7.34 ; \text { sig } \\
\text { Controls: } 39.13 \pm 7.74 ; \mathrm{sig}\end{array}$ & $\begin{array}{l}\text { Cases: } 5.41 \pm 0.92 \\
\text { Controls: } 4.41 \pm 0.98 \\
\text { Sleep anxiety } \\
\text { Cases: } 7.73 \pm 2.59 \\
\text { Controls: } 4.41 \pm 0.80 \\
\text { Night wakings } \\
\text { Cases: } 5.59 \pm 1.61 \\
\text { Controls: } 4.11 \pm 1.39 \\
\text { Parasomnias } \\
\text { Cases: } 9.80 \pm 2.52 \\
\text { Controls: } 8.51 \pm 1.76 \\
\text { Sleep-disordered breathing } \\
\text { Cases: } 3.63 \pm 0.73 \\
\text { Controls: } 3.59 \pm 0.90 \\
\text { Daytime sleepiness } \\
\text { Cases: } 10.44 \pm 1.84 \\
\text { Controls: } 9.89 \pm 1.85 \\
\text { Total sleep time } \\
\text { Cases: } 8.16 \pm 0.89 \\
\text { Controls: } 9.11 \pm 0.89\end{array}$ & $\begin{array}{l}\text { Sleep duration } \\
\text { Cases: } 4.70 \pm 0.95 \text {; sig } \\
\text { Controls: } 4.18 \pm 0.90 \text {; sig } \\
\text { Sleep anxiety } \\
\text { Cases: } 6.78 \pm 2.35 \text {; sig } \\
\text { Controls: } 4.05 \pm 0.4 \text {; sig } \\
\text { Night wakings } \\
\text { Cases: } 4.24 \pm 1.59 \text {; sig } \\
\text { Controls: } 3.32 \pm 0.62 \text {; sig } \\
\text { Parasomnias } \\
\text { Cases: } 8.75 \pm 1.84 \text {; sig } \\
\text { Controls: } 7.16 \pm 0.44 \text {; ig } \\
\text { Sleep-disordered breathing } \\
\text { Cases: } 3.41 \pm 059 \text {; sig } \\
\text { Controls: } 3.40 \pm 0.76 \text {; sig } \\
\text { Daytime sleepiness } \\
\text { Cases: } 10.17 \pm 1.93 \text {; NS } \\
\text { Controls: } 9.21 \pm 1.08 \text {; sig } \\
\text { Total sleep time } \\
\text { Cases: } 8.63 \pm 0.85 \text {; sig } \\
\text { Controls: } 9.29 \pm 0.89 \text {; sig }\end{array}$ & $\begin{array}{l}\text { VDS may be beneficial in ASD patients } \\
\text { and healthy individuals with } \\
\text { sleep disturbances }\end{array}$ \\
\hline Arico [38], Italy & RLS severity: IRLS-RS & 10.3 & $\begin{array}{l}30.4 \text { (no information on } \\
\text { statistical significance) }\end{array}$ & 19.8 & $\begin{array}{l}8.6 \text { (no information on statistical } \\
\text { significance) }\end{array}$ & $\begin{array}{l}\text { VDS has a therapeutic effect in } \\
\text { decreasing RLS severity }\end{array}$ \\
\hline \multicolumn{7}{|l|}{ Sleep quality } \\
\hline Huang [42], USA & $\begin{array}{l}\text { Sleep quality: PSQI } \\
\text { Sleep latency: \#2 "how long } \\
\text { has it usually taken you to } \\
\text { fall asleep each night" of } \\
\text { the PSQI } \\
\text { Sleep duration: \#4 "how } \\
\text { many hours of actual sleep } \\
\text { did you get at night" of } \\
\text { the PSQI } \\
\text { Sleep efficiency: \#4+\#1 } \\
\text { "what time have you usually } \\
\text { gone to bed at night" + \#3 } \\
\text { "what time have you usually } \\
\text { gotten up in the morning" of } \\
\text { the PSQI }\end{array}$ & $\begin{array}{l}\text { Total: } 18.57 \pm 5.42 \\
\text { INS: } 22.73 \pm 1.83 \\
\text { DEF: } 13.77 \pm 3.94\end{array}$ & $\begin{array}{l}\text { Total: } 26.00 \pm 8.38 \text { (sig. } \\
\text { increase) } \\
\text { INS: } 29.60 \pm 11.67 \\
\text { DEF: } 24.00 \pm 5.79\end{array}$ & $\begin{array}{l}\text { Global PSQI score } \\
\text { Total: } 13.46 \pm 4.92 \\
\text { INS: } 12.27 \pm 5.55 \\
\text { DEF: } 14.85 \pm 3.83 \\
\text { Sleep latency (min) } \\
\text { Total: } 67.22 \pm 56.13 \\
\text { INS: } 41.61 \pm 48.21 \\
\text { DEF: } 94.81 \pm 52.15 \\
\text { Sleep duration (h) } \\
\text { Total: } 4.59 \pm 1.84 \\
\text { INS: } 5.33 \pm 1.88 \\
\text { DEF: } 3.73 \pm 1.41 \\
\text { Sleep efficiency (\%) } \\
\text { Total: } 59.79 \pm 25.31 \\
\text { INS: } 66.97 \pm 23.7 \\
\text { DEF: } 52.61 \pm 25.70\end{array}$ & $\begin{array}{l}\text { Global PSQI score } \\
\text { Total: } 12.22 \pm 4.61 \text { (sig. decrease) } \\
\text { INS: } 11.29 \pm 4.66 \\
\text { DEF: } 13.23 \pm 4.51 \text { (sig. decrease) } \\
\text { Sleep latency (min) } \\
\text { Total: } 57.86 \pm 44.03 \text { (sig. decrease) } \\
\text { INS: } 39.83 \pm 39.05 \\
\text { DEF: } 78.65 \pm 41.33 \text { (sig. decrease) } \\
\text { Sleep duration (h) } \\
\text { Total: } 5.30 \pm 1.57 \text { (sig. decrease) } \\
\text { INS: } 5.90 \pm 1.55 \text { (sig. decrease) } \\
\text { DEF: } 4.62 \pm 1.33 \text { (sig. decrease) } \\
\text { Sleep efficiency (\%) } \\
\text { Total: } 66.62 \pm 18.61 \text { (sig. increase) } \\
\text { INS: } 70.34 \pm 17.76 \\
\text { DEF: } 62.33 \pm 19.34 \text { (sig. increase) }\end{array}$ & $\begin{array}{l}\text { Sig. improvement in overall sleep } \\
\text { quality, sleep latency, sleep duration, and } \\
\text { sleep efficiency in veterans with multiple } \\
\text { areas of chronic pain with VDS (after } \\
\text { controlling for potential confounders } \\
\text { improvement in sleep efficiency became } \\
\text { borderline significant) } \\
\text { The magnitudes of sleep improvement in } \\
\text { latency, duration, and efficiency were all } \\
\text { larger in the DEF subgroup; however, } \\
\text { the difference in improvements between } \\
\text { the subgroups was NS except for } \\
\text { sleep latency }\end{array}$ \\
\hline
\end{tabular}

I: intervention; C: control; PSQI: Pittsburgh Sleep Quality Index; NR: not reported; INS: insufficient; DEF: deficient; VDS: vitamin D supplementation; 25(OH)D: 25-hydroxyvitamin D SD: Standard Deviation; NS: Not Significant; sig. Significant; IU: International Unit; ESS: Epworth Sleepiness Scale; NR: not reported; RLS: restless legs syndrome; IRLSSG: International Restless Legs Syndrome Study Group; CI: confidence interval; IRLS-RS: International Restless Legs Syndrome Rating Scale; ADHD: Attention Deficit Hyperactivity Disorder; IQR Interquartile Range; ASD: Autism Spectrum Disorder; CSHQ: Children's Sleep Habits Questionnaire IRLS-RS: International Restless Legs Syndrome Rating Scale; IRLSSG: Internationa Restless Legs Syndrome Study Group; CSHQ: Children's Sleep Habits Questionnaire; PHQ-8 Item: The 8 item Patient Health Questionnaire depression scale; NA: Not Applicable; SE: Standard Error. 


\subsection{Sleep Quality}

The pre-post studies by Huang et al. [42], Eshaghi et al. [39], and Maheshwari et al. [41] investigated the effect of VDS on sleep quality assessed by the PSQI. All three trials showed a significant improvement in overall sleep quality with VDS. However, the three trials were heterogeneous and did not contain ample information allowing pooling; hence, performing a meta-analysis of their results was impossible.

The four RCTs conducted by Ghaderi et al. [25], Majid et al. [27], Mason et al. [28], and Mirzaei et al. [30] explored the effect of VDS on sleep quality assessed by the PSQI. Only three $[25,27,30]$ provided information and had similar characteristics to allow pooling, whereas the study by Mason et al. [28] did not report on numerical outcomes, and therefore was not included in the meta-analysis. This study showed no significant change in overall sleep quality with VDS and a deterioration in total PSQI among women who repleted their vitamin D levels, concluding that VDS of $2000 \mathrm{IU} / \mathrm{d}$ may result in overall worse sleep quality for postmenopausal women with low circulating vitamin D undergoing weight loss.

As for the results of the meta-analysis of the eligible RCTs $[25,27,30]$, the forest plot for the mean difference in the PSQI based on VDS is presented in Figure 3. The three studies included patients undergoing maintenance methadone treatment [25], people with a PSQI $\geq 5$ [27], and fibromyalgia syndrome patients [30]. The duration of VDS was short $(8[27,30]$ to 12 weeks [25]), and the dose was either 3571.42 [25,27] or 7142.85 IU [30]. A statistically significant decrease in the PSQI in the group receiving VDS as compared with placebo was shown by the meta-analysis (mean difference, -2.33 (95\% CI, $-3.09,-1.57$ ); $p<0.001)$. The heterogeneity of the analysis was null $\left(\mathrm{I}^{2}=0 \%\right)$. The overall certainty of the evidence of the meta-analysis was moderate (Supplementary S2).

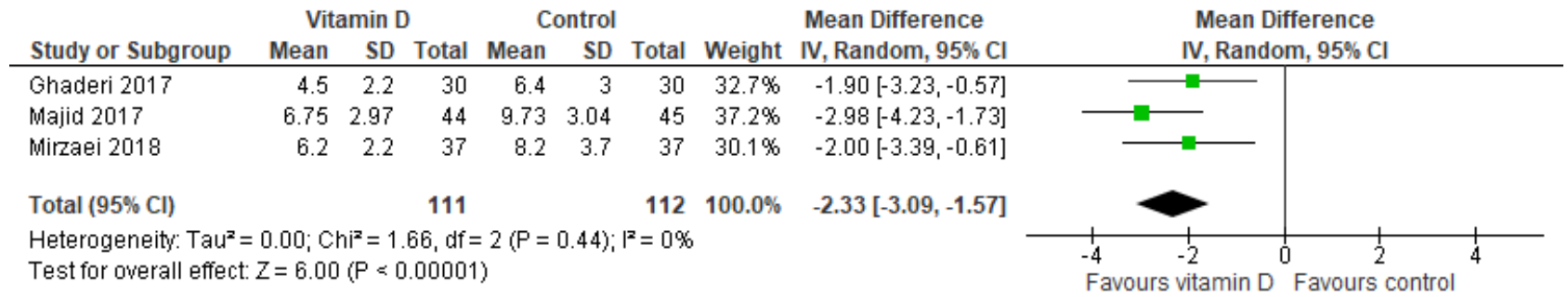

Figure 3. Meta-analysis of the effect of vitamin D supplementation on sleep quality assessed by the Pittsburg Sleep Quality Index. Mean differences for each study are represented by squares, and 95\% Confidence Intervals are represented by the lines through the squares. The pooled mean differences are represented by diamonds. Between-study heterogeneity was assessed with the use of the $\mathrm{I}^{2}$ statistic $[25,27,30]$.

\subsection{Other Outcomes}

\section{Disturbed sleeping}

The only pre-post study [40] investigating sleep habits and disorders in children with ASD showed that VDS may be beneficial in these patients, as well as healthy individuals with sleep disturbances.

As for RCTs, the effects of VDS on sleep-related impairment, sleep difficulty, and sleep disorders were assessed by McCarthy et al. [29], Okereke et al. [32], and Zhu et al. [36], respectively; the results were not unanimous. While McCarthy et al. [29] showed a statistically significant improvement in sleep-related impairment with VDS, Okereke et al. [32] and Zhu et al. [36] did not report on such findings. In both RCTs, there were non-significant differences in likelihood of sleep problems with VDS compared with placebo after controlling for confounding variables). 


\section{Sleepiness}

Two RCTs assessed the effect of VDS on sleepiness using different tools [26,34]. The study of Kerley et al. [26], which was conducted on patients with OSA, did not report any difference in sleepiness between the group receiving VDS and those receiving placebo. In contrast, the study by Sharifan et al. [34], which was conducted on patients with insomnia, showed a beneficial effect of vitamin D3-fortified low-fat milk on insomnia symptoms compared with unfortified milk. No effect was detected with vitamin D3-fortified low-fat yogurt compared with unfortified one.

\section{RLS}

The pre-post study conducted by Arico et al. [38] found that long-term VDS (6 months) decreased RLS severity in a small population (5 patients). In contrast, the RCT by Wali et al. [35] found no effect of VDS on severity of RLS compared with placebo.

\subsection{Sleep Problems as Adverse Events of VDS}

Sleep problems as an adverse event of VDS were assessed in the two RCTs conducted by Mohammadpour et al. [31], and de Koning et al. [24]. Both studies showed no significant difference in sleep problems with VDS versus placebo in children with ADHD or community-dwelling people with depressive symptoms, respectively.

\section{Discussion}

This systematic review and meta-analysis investigated the effect of VDS on sleep quantity and quality, and sleep disorders. VDD is an emerging risk factor for suboptimal sleep and sleep disorders $[12,15,43]$. Such an association has been observed in several healthy and ill populations including factory workers, older community-dwelling men, hemodialysis patients, and pregnant women [12]. Specifically, through a meta-analysis of observational studies involving 9397 participants, Gao et al. found that participants with VDD had increased odds of sleep disorders and poor sleep quality by 1.5 fold, short sleep duration by 1.75 fold, and sleepiness by 1.36 fold. They also provided evidence that serum $25(\mathrm{OH}) \mathrm{D}$ below $20 \mathrm{ng} / \mathrm{mL}$ could significantly heighten the odds of unhealthy sleep [15]. Similarly, through a meta-analysis of observational studies conducted on 1864 subjects with sleep disorders and 1340 control participants, Yan et al. [43] found that the average serum vitamin D concentration in the group with sleep disorders was $0.75 \mathrm{ng} / \mathrm{mL}$ lower than that in the control group [43].

The association between VDS and sleep regulation is biologically plausible and worth investigation given its clinical and public health relevance. Nevertheless, we found a limited number of human interventional studies-especially RCTs—and most of the included studies focused on sleep quality compared with other sleep-related outcomes. The evidence from included studies was promising regarding the effectiveness of VDS on enhancing sleep quality; nevertheless, studies investigating sleep quantity and sleep disorders were scarce, heterogeneous in terms of included populations and methodologies, and their findings were not unanimous, preventing generating a solid conclusion. Hence, our results suggest that VDS is promising in improving sleep quality; however, its effect on sleep quantity and disorders needs to be further investigated.

Although the exact physiological mechanisms between vitamin $\mathrm{D}$ and sleep regulation have not yet been fully uncovered, several direct and indirect mechanisms have been suggested [12]. One potential mechanism is the extensive presence of vitamin D receptors in many parts of the brain, specifically areas that affect sleep [44]. Another theory involves the expression enzymes involved in vitamin D activation and degradation (25-hydroxylase and 1-hydroxylase and 24-CYP24A1) in areas in the brain known to be involved in sleep regulation including the supraoptic and paraventricular nuclei within the hypothalamus and the substantia nigra [44,45]. Another plausible theory considers the effect of sunlight. It is well known that vitamin D levels are regulated by exposure to sunlight and since sunlight also affects the circadian rhythm, it is highly plausible to assume that there 
is a link between those factors [46-49]. Furthermore, the production of melatonin-a hormone involved in the regulation of circadian rhythms and sleep-is regulated by vitamin D; thus, impaired vitamin D levels could decrease melatonin levels, potentially leading to sleep disorders [50,51]. One final plausible mechanism is that vitamin D, as an immunomodulatory molecule, plays a role in downregulating inflammatory markers that are involved in sleep regulation such as tumor necrosis factor $\alpha$ (TNF- $\alpha)$, cytokines and prostaglandin D2. In the case of VDD, such inflammatory markers would be raised, negatively affecting sleep $[52,53]$. All of these factors may explain our findings regarding the beneficial effect of VDS on sleep quality.

The only study involving patients with OSA was conducted by Kerley et al. [26]. VDD is a common finding in OSA patients compared with non-apneic subjects, and vitamin D levels were shown to be inversely correlated with the severity of OSA [54]. This association is likely to be mediated by complex pathogenetic mechanisms, such as immune system modulation, myopathy, and inflammation; nevertheless, these mechanisms are not fully understood yet. Additionally, this relationship seems to be confounded by numerous factors, such as obesity [54]. Kerley et al. [26] did not find a beneficial effect of VDS on improving sleepiness in this patient population. This might be due to the small sample of the trial and to the fact that $90 \%$ of the VDS group were stable on continuous positive airways pressure, which may have diluted any potential benefit of VDS [26].

Our findings concerning the effectiveness of VDS on RLS were contradictory [35,38]. VDD may play a role in this movement disorder through its link with dopaminergic dysfunction. Nevertheless, to date, the causality between VDD and RLS is only hypothesized. This null effect, despite the significant improvement in vitamin D levels in the intervention group, suggests that VDS may not have a therapeutic effect in RLS, although it may contribute to the pathophysiology of the syndrome [35]. The authors of this study argue that in RLS, vitamin D levels in the brain are more important than in the blood. Accordingly, the improvement in serum levels with VDS may not have sufficiently affected vitamin D levels in the brain [35].

It should be noted that vitamin D levels in response to supplementation depend on three factors, dose, frequency and interval [55]; accordingly, our findings could be interpreted in light of this fact. For example, the RCTs that found a beneficial effect of VDS on sleep disorders were conducted over a short period of time, i.e., 10 weeks in the study by McCarthy et al. [29], and 12 weeks in the studies by Rorie et al. [33] and Sharifan et al. [34]. In contrast, RCTs reporting no significant improvement in sleep disorders with VDS were those conducted over a long duration, i.e., 6 months in the study by Zhu et al. [36], 18 months in the study by Slow et al. [37], and a median follow-up duration of 5.3 years in the study by Okereke et al. [32]. This observation, although it could be attributed to seasonality, raises queries on whether extended supplementation with vitamin D may not always result in better outcomes.

Finally, human circadian rhythms—sleep and wakefulness cycles—are synchronized by environmental signals, especially light and dark intervals through sunlight [48]. Although intentional sun exposure could hence be recommended to enhance sleep and vitamin D levels, it remains highly challenging to titrate one's exposure, besides the documented negative side effects of ultraviolet irradiation.

\section{Strengths and Limitations}

Our study has numerous strengths. First, we followed a systematic approach in our search and analysis, using a highly sensitive search strategy, and followed recommended reporting approaches for the review [23] as well as the search strategy [22]. Second, we contacted the authors of some included studies to obtain additional data when they were not reported in the published studies. Unfortunately, we did not receive feedback from all authors. Finally, the risk of bias of the majority of included studies was low.

Nevertheless, the current analysis had some limitations. First, we were limited to study level rather than individual-level data, which would have been more accurate than 
the overall mean change in sleep. Second, there were variabilities between included studies, which complicates the comparisons as well as the interpretation of our results, especially in the study populations, the outcomes assessed, and the assessment methods. Third, some papers did not provide crucial information such as the form of vitamin D, the levels of vitamin $\mathrm{D}$ at the end of the study, the daily dose equivalent, the assessment method of vitamin D levels, nor compliance with VDS. Although we contacted respective authors, we could not obtain the needed information in some instances. This would have enabled us to better interpret our findings. Fourth, while we tried to make the literature search as exhaustive as possible, pertinent studies might have been missed; a common limitation to systematic reviews. Furthermore, we did not have access to some potentially eligible studies for full-text screening. Fifth, given the small number of studies investigating the effect of VDS on sleep disorders and their heterogeneity, we could not perform a metaanalysis of their findings and our conclusion remains limited to a qualitative synthesis. Finally, repeat screening, selection of studies, data extraction, and quality assessment was not possible.

\section{Conclusions}

In conclusion, the evidence presented in this review suggests a beneficial role of VDS in enhancing sleep quality. These results remain to be interpreted with caution given the limited number of included RTCs and their relatively small sample size. Nevertheless, the positive effects of such supplementation could be considered in clinical practice, especially in the context of beneficial skeletal [56] and pleiotropic extraskeletal effects $[57,58]$ of vitamin D, as well as the relatively limited cost of VDS. As we could not find enough studies assessing the effect of VDS on sleep disorders, OSA, sleepiness, and RLS, this remains to be explored in future adequately powered, high-quality RCTs.

Supplementary Materials: The following supporting information can be downloaded at: https: / /www.mdpi.com/article/10.3390/nu14051076/s1, S1,S2: Search strategy.

Funding: Funded by Cluster Grant R18030. College of Natural and Health Sciences, Zayed University, Dubai, United Arab Emirates. The funding body was not involved in study design, data collection and analysis, results interpretation, and manuscript write up.

Institutional Review Board Statement: Not applicable.

Informed Consent Statement: Not applicable.

Conflicts of Interest: The author declare no conflict of interest.

\section{References}

1. Hillman, D.; Mitchell, S.; Streatfeild, J.; Burns, C.; Bruck, D.; Pezzullo, L. The economic cost of inadequate sleep. Sleep 2018, 41, zsy083. [CrossRef]

2. Skaer, T.L.; Sclar, D.A. Economic implications of sleep disorders. Pharmacoeconomics 2010, 28, 1015-1023. [CrossRef] [PubMed]

3. Sateia, M.J. International classification of sleep disorders-third edition: Highlights and modifications. Chest 2014, 146, 1387-1394. [CrossRef] [PubMed]

4. Coren, S. The prevalence of self-reported sleep disturbances in young adults. Int. J. Neurosci. 1994, 79, 67-73. [CrossRef] [PubMed]

5. Luyster, F.S.; Strollo, P.J., Jr.; Zee, P.C.; Walsh, J.K. Sleep: A health imperative. Sleep 2012, 35, 727-734. [CrossRef] [PubMed]

6. Irwin, M.R. Why sleep is important for health: A psychoneuroimmunology perspective. Annu. Rev. Psychol. 2015, 66, 143-172. [CrossRef]

7. Chokroverty, S. Overview of sleep \& sleep disorders. Indian J. Med. Res. 2010, 131, 126-140.

8. Institute of Medicine, Committee on Sleep Medicine and Research. The National Academies Collection: Reports funded by National Institutes of Health. In Sleep Disorders and Sleep Deprivation: An Unmet Public Health Problem; Colten, H.R., Altevogt, B.M., Eds.; National Academy of Sciences: Washington, DC, USA, 2006.

9. Liew, S.C.; Aung, T. Sleep deprivation and its association with diseases-A review. Sleep Med. 2021, 77, 192-204. [CrossRef]

10. Andersen, M.L.; Tufik, S. Vitamin D as an underlying factor in sleep-related issues. J. Clin. Sleep Med. JCSM Off. Publ. Am. Acad. Sleep Med. 2012, 8, 699. [CrossRef]

11. Anglin, R.E.; Samaan, Z.; Walter, S.D.; McDonald, S.D. Vitamin D deficiency and depression in adults: Systematic review and meta-analysis. Br. J. Psychiatry J. Ment. Sci. 2013, 202, 100-107. [CrossRef] 
12. Romano, F.; Muscogiuri, G.; Di Benedetto, E.; Zhukouskaya, V.V.; Barrea, L.; Savastano, S.; Colao, A.; Di Somma, C. Vitamin D and Sleep Regulation: Is there a Role for Vitamin D? Curr. Pharm. Des. 2020, 26, 2492-2496. [CrossRef] [PubMed]

13. Muscogiuri, G.; Barrea, L.; Scannapieco, M.; Di Somma, C.; Scacchi, M.; Aimaretti, G.; Savastano, S.; Colao, A.; Marzullo, P. The lullaby of the sun: The role of vitamin D in sleep disturbance. Sleep Med. 2019, 54, 262-265. [CrossRef]

14. Al-Shawwa, B.; Ehsan, Z.; Ingram, D.G. Vitamin D and sleep in children. J. Clin. Sleep Med. JCSM Off. Publ. Am. Acad. Sleep Med. 2020, 16, 1119-1123. [CrossRef] [PubMed]

15. Gao, Q.; Kou, T.; Zhuang, B.; Ren, Y.; Dong, X.; Wang, Q. The Association between Vitamin D Deficiency and Sleep Disorders: A Systematic Review and Meta-Analysis. Nutrients 2018, 10, 1395. [CrossRef] [PubMed]

16. Stumpf, W.E.; Bidmon, H.J.; Li, L.; Pilgrim, C.; Bartke, A.; Mayerhofer, A.; Heiss, C. Nuclear receptor sites for vitamin D-soltriol in midbrain and hindbrain of Siberian hamster (Phodopus sungorus) assessed by autoradiography. Histochemistry 1992, 98, 155-164. [CrossRef] [PubMed]

17. Stumpf, W.E.; O'Brien, L.P. 1,25 (OH $)_{2}$ vitamin D3 sites of action in the brain. An autoradiographic study. Histochemistry 1987, 87, 393-406. [CrossRef]

18. Patrick, R.P.; Ames, B.N. Vitamin D and the omega-3 fatty acids control serotonin synthesis and action, part 2: Relevance for ADHD, bipolar disorder, schizophrenia, and impulsive behavior. FASEB J. Off. Publ. Fed. Am. Soc. Exp. Biol. 2015, 29, $2207-2222$. [CrossRef]

19. Thorpy, M. International classification of sleep disorders. In Sleep Disorders Medicine; Springer: Berlin/Heidelberg, Germany, 2017; pp. $475-484$.

20. Higgins, J.P.T.; Savović, J.; Page, M.J.; Elbers, R.G.; Sterne, J.A.C. Chapter 8: Assessing risk of bias in a randomized trial. In Cochrane Handbook for Systematic Reviews of Interventions Version 6.0; Higgins, J.P.T., Thomas, J., Chandler, J., Cumpston, M., Li, T., Page, M.J., Welch, V.A., Eds.; Cochrane: Chichester, UK, 2019.

21. Guyatt, G.H.; Oxman, A.D.; Vist, G.; Kunz, R.; Brozek, J.; Alonso-Coello, P.; Montori, V.; Akl, E.A.; Djulbegovic, B.; Falck-Ytter, Y.; et al. GRADE guidelines: 4. Rating the quality of evidence-study limitations (risk of bias). J. Clin. Epidemiol. 2011, 64, 407-415. [CrossRef]

22. Rethlefsen, M.L.; Kirtley, S.; Waffenschmidt, S.; Ayala, A.P.; Moher, D.; Page, M.J.; Koffel, J.B. PRISMA-S: An extension to the PRISMA Statement for Reporting Literature Searches in Systematic Reviews. Syst. Rev. 2021, 10, 39. [CrossRef]

23. Page, M.J.; Moher, D.; Bossuyt, P.M.; Boutron, I.; Hoffmann, T.C.; Mulrow, C.D.; Shamseer, L.; Tetzlaff, J.M.; Akl, E.A.; Brennan, S.E.; et al. PRISMA 2020 explanation and elaboration: Updated guidance and exemplars for reporting systematic reviews. BMJ 2021, 372, n160. [CrossRef]

24. De Koning, E.J.; Lips, P.; Penninx, B.; Elders, P.J.M.; Heijboer, A.C.; den Heijer, M.; Bet, P.M.; van Marwijk, H.W.J.; van Schoor, N.M. Vitamin D supplementation for the prevention of depression and poor physical function in older persons: The D-Vitaal study, a randomized clinical trial. Am. J. Clin. Nutr. 2019, 110, 1119-1130. [CrossRef] [PubMed]

25. Ghaderi, A.; Banafshe, H.R.; Motmaen, M.; Rasouli-Azad, M.; Bahmani, F.; Asemi, Z. Clinical trial of the effects of vitamin D supplementation on psychological symptoms and metabolic profiles in maintenance methadone treatment patients. Prog. Neuro-Psychopharmacol. Biol. Psychiatry 2017, 79 Pt B, 84-89. [CrossRef]

26. Kerley, C.P.; Hutchinson, K.; Bramham, J.; McGowan, A.; Faul, J.; Cormican, L. Vitamin D Improves Selected Metabolic Parameters but Not Neuropsychological or Quality of Life Indices in OSA: A Pilot Study. J. Clin. Sleep Med. JCSM Off. Publ. Am. Acad. Sleep Med. 2017, 13, 19-26. [CrossRef] [PubMed]

27. Majid, M.S.; Ahmad, H.S.; Bizhan, H.; Hosein, H.Z.M.; Mohammad, A. The effect of vitamin D supplement on the score and quality of sleep in 20-50 year-old people with sleep disorders compared with control group. Nutr. Neurosci. 2018, 21, 511-519. [CrossRef] [PubMed]

28. Mason, C.; de Dieu Tapsoba, J.; Duggan, C.; Wang, C.Y.; Korde, L.; McTiernan, A. Repletion of vitamin D associated with deterioration of sleep quality among postmenopausal women. Prev. Med. 2016, 93, 166-170. [CrossRef]

29. McCarthy, M.S.; Elshaw, E.B.; Szekely, B.M.; Raju, D. A Prospective Cohort Study of Vitamin D Supplementation in AD Soldiers: Preliminary Findings. Mil. Med. 2019, 184 (Suppl. S1), 498-505. [CrossRef]

30. Mirzaei, A.; Zabihiyeganeh, M.; Jahed, S.A.; Khiabani, E.; Nojomi, M.; Ghaffari, S. Effects of vitamin D optimization on quality of life of patients with fibromyalgia: A randomized controlled trial. Med. J. Islamic Repub. Iran 2018, 32, 29. [CrossRef]

31. Mohammadpour, N.; Jazayeri, S.; Tehrani-Doost, M.; Djalali, M.; Hosseini, M.; Effatpanah, M.; Davari-Ashtiani, R.; Karami, E. Effect of vitamin D supplementation as adjunctive therapy to methylphenidate on ADHD symptoms: A randomized, double blind, placebo-controlled trial. Nutr. Neurosci. 2018, 21, 202-209. [CrossRef]

32. Okereke, O.I.; Reynolds, C.F., 3rd; Mischoulon, D.; Chang, G.; Vyas, C.M.; Cook, N.R.; Weinberg, A.; Bubes, V.; Copeland, T.; Friedenberg, G.; et al. Effect of Long-term Vitamin D3 Supplementation vs Placebo on Risk of Depression or Clinically Relevant Depressive Symptoms and on Change in Mood Scores: A Randomized Clinical Trial. JAMA 2020, 324, 471-480. [CrossRef]

33. Rorie, A.; Goldner, W.S.; Lyden, E.; Poole, J.A. Beneficial role for supplemental vitamin D3 treatment in chronic urticaria: A randomized study. Ann. Allergy Asthma Immunol. Off. Publ. Am. Coll. Allergy Asthma Immunol. 2014, 112, 376-382. [CrossRef]

34. Sharifan, P.; Khoshakhlagh, M.; Khorasanchi, Z.; Darroudi, S.; Rezaie, M.; Safarian, M.; Vatanparast, H.; Afshari, A.; Ferns, G.; Ghazizadeh, H.; et al. Efficacy of low-fat milk and yogurt fortified with encapsulated vitamin D3 on improvement in symptoms of insomnia and quality of life: Evidence from the SUVINA trial. Food Sci. Nutr. 2020, 8, 4484-4490. [CrossRef] [PubMed] 
35. Wali, S.O.; Abaalkhail, B.; Alhejaili, F.; Pandi-Perumal, S.R. Efficacy of vitamin D replacement therapy in restless legs syndrome: A randomized control trial. Sleep Breath. Schlaf Atm. 2019, 23, 595-601. [CrossRef]

36. Zhu, C.; Zhang, Y.; Wang, T.; Lin, Y.; Yu, J.; Xia, Q.; Zhu, P.; Zhu, D.M. Vitamin D supplementation improves anxiety but not depression symptoms in patients with vitamin D deficiency. Brain Behav. 2020, 10, e01760. [CrossRef] [PubMed]

37. Slow, S.; Florkowski, C.M.; Chambers, S.T.; Priest, P.C.; Stewart, A.W.; Jennings, L.C.; Livesey, J.H.; Camargo, C.A., Jr.; Scragg, R.; Murdoch, D.R. Effect of monthly vitamin D3 supplementation in healthy adults on adverse effects of earthquakes: Randomised controlled trial. BMJ 2014, 349, g7260. [CrossRef] [PubMed]

38. Aricò, I.; Campolo, L.; Silvestri, R. Preliminary Data on Vitamin D Deficiency and Treatment in a Cohort of Sicilian RLS/WED Patients. Neuropsychiatr. Dis. Treat 2014, 10, 953-958.

39. Eshaghi, S.; Morteza, T.; Khadijeh, I.; Knechtle, B.; Nikolaidis, P.T.; Chtourou, H. The effect of aerobic training and vitamin D supplements on the neurocognitive functions of elderly women with sleep disorders. Biol. Rhythm. Res. 2020, 51, 727-734. [CrossRef]

40. Guler, S.; Yeşil, G.; Önal, H.; Ekici, B.; Ozdil, M. Sleep disturbances and serum vitamin D levels in children with autism spectrum disorder. Int. J. Clin. Exp. Med. 2016, 9, 14691-14697.

41. Maheshwari, P.; Arun, S.; Venkatesh, N.; Sushmi, K.; Shanmugarajan, T.; Shanmugasundaram, P. Effect of Vitamin D supplement in improving pain, sleep, and quality of life on patients with chronic low back pain. Drug Invent. Today 2019, 12, 2508-2510.

42. Huang, W.; Shah, S.; Long, Q.; Crankshaw, A.K.; Tangpricha, V. Improvement of pain, sleep, and quality of life in chronic pain patients with vitamin D supplementation. Clin. J. Pain 2013, 29, 341-347. [CrossRef]

43. Yan, S.; Tian, Z.; Zhao, H.; Wang, C.; Pan, Y.; Yao, N.; Guo, Y.; Wang, H.; Li, B.; Cui, W. A meta-analysis: Does vitamin D play a promising role in sleep disorders? Food Sci. Nutr. 2020, 8, 5696-5709. [CrossRef]

44. Eyles, D.W.; Smith, S.; Kinobe, R.; Hewison, M.; McGrath, J.J. Distribution of the vitamin D receptor and 1 alpha-hydroxylase in human brain. J. Chem. Neuroanat. 2005, 29, 21-30. [CrossRef] [PubMed]

45. Garcion, E.; Wion-Barbot, N.; Montero-Menei, C.N.; Berger, F.; Wion, D. New clues about vitamin D functions in the nervous system. Trends Endocrinol. Metab. TEM 2002, 13, 100-105. [CrossRef]

46. Lucock, M.; Jones, P.; Martin, C.; Beckett, E.; Yates, Z.; Furst, J.; Veysey, M. Vitamin D: Beyond Metabolism. J. Evid. Based Complement. Altern. Med. 2015, 20, 310-322. [CrossRef] [PubMed]

47. Vitaterna, M.H.; Takahashi, J.S.; Turek, F.W. Overview of circadian rhythms. Alcohol Res. Health J. Natl. Inst. Alcohol Abus. Alcohol. 2001, 25, 85-93.

48. Dibner, C.; Schibler, U.; Albrecht, U. The mammalian circadian timing system: Organization and coordination of central and peripheral clocks. Annu. Rev. Physiol. 2010, 72, 517-549. [CrossRef]

49. Jamilian, H.; Amirani, E.; Milajerdi, A.; Kolahdooz, F.; Mirzaei, H.; Zaroudi, M.; Ghaderi, A.; Asemi, Z. The effects of vitamin D supplementation on mental health, and biomarkers of inflammation and oxidative stress in patients with psychiatric disorders: A systematic review and meta-analysis of randomized controlled trials. Prog. Neuro-Psychopharmacol. Biol. Psychiatry 2019, 94, 109651. [CrossRef]

50. Zhao, D.; Yu, Y.; Shen, Y.; Liu, Q.; Zhao, Z.; Sharma, R.; Reiter, R.J. Melatonin Synthesis and Function: Evolutionary History in Animals and Plants. Front. Endocrinol. 2019, 10, 249. [CrossRef]

51. Kaneko, I.; Sabir, M.S.; Dussik, C.M.; Whitfield, G.K.; Karrys, A.; Hsieh, J.C.; Haussler, M.R.; Meyer, M.B.; Pike, J.W.; Jurutka, P.W. 1,25-Dihydroxyvitamin D regulates expression of the tryptophan hydroxylase 2 and leptin genes: Implication for behavioral influences of vitamin D. FASEB J. Off. Publ. Fed. Am. Soc. Exp. Biol. 2015, 29, 4023-4035. [CrossRef]

52. Jablonski, K.L.; Chonchol, M.; Pierce, G.L.; Walker, A.E.; Seals, D.R. 25-Hydroxyvitamin D deficiency is associated with inflammation-linked vascular endothelial dysfunction in middle-aged and older adults. Hypertension 2011, 57, 63-69. [CrossRef]

53. Krueger, J.M.; Majde, J.A.; Rector, D.M. Cytokines in immune function and sleep regulation. Handb. Clin. Neurol. 2011, 98, 229-240.

54. Archontogeorgis, K.; Nena, E.; Papanas, N.; Steiropoulos, P. The role of vitamin D in obstructive sleep apnoea syndrome. Breathe 2018, 14, 206-215. [CrossRef]

55. Chao, Y.S.; Brunel, L.; Faris, P.; Veugelers, P.J. The importance of dose, frequency and duration of vitamin D supplementation for plasma 25-hydroxyvitamin D. Nutrients 2013, 5, 4067-4078. [CrossRef]

56. Chakhtoura, M.; Chamoun, N.; Rahme, M.; Fuleihan, G.E. Impact of vitamin D supplementation on falls and fractures-A critical appraisal of the quality of the evidence and an overview of the available guidelines. Bone 2020, 131, 115112. [CrossRef] [PubMed]

57. Caprio, M.; Infante, M.; Calanchini, M.; Mammi, C.; Fabbri, A. Vitamin D: Not just the bone. Evidence for beneficial pleiotropic extraskeletal effects. Eat. Weight Disord. EWD 2017, 22, 27-41. [CrossRef] [PubMed]

58. Egierska, D.; Pietruszka, P.; Burzyńska, P.; Chruścicka, I.; Buchta, J. Pleiotropic effects of vitamin D3. J. Educ. Health Sport 2021, 11, 143-155. [CrossRef] 